TRANSACTIONS OF THE

AMERICAN MATHEMATICAL SOCIETY

Volume 361, Number 12, December 2009, Pages 6475-6500

S 0002-9947(09)04839-9

Article electronically published on July 20, 2009

\title{
A PRIORI ESTIMATES FOR A CLASS OF QUASI-LINEAR ELLIPTIC EQUATIONS
}

\author{
DANIEL DANERS AND PAVEL DRÁBEK
}

\begin{abstract}
In this paper we prove a priori estimates for a class of quasi-linear elliptic equations. To make the proofs clear and transparent we concentrate on the $p$-Laplacian. We focus on $L_{p}$-estimates for weak solutions of the problem with all standard boundary conditions on non-smooth domains. As an application we prove existence, continuity and compactness of the resolvent operator. We finally prove estimates for solutions to equations with non-linear source and show that, under suitable growth conditions, all solutions are globally bounded.
\end{abstract}

\section{INTRODUCTION}

The purpose of this paper is to prove global a priori estimates for weak solutions to the $p$-Laplace equation

$$
\begin{array}{rlrl}
-\Delta_{p} u+c_{0}|u|^{p-2} u & =f & & \text { in } \Omega, \\
\mathcal{B} u=0 & & \text { on } \partial \Omega
\end{array}
$$

on an open set $\Omega \subset \mathbb{R}^{N}$. Here $\Delta_{p} u:=\operatorname{div}\left(|\nabla u|^{p-2} \nabla u\right)$ is the $p$-Laplacian with $p \in(1, \infty)$, and $\mathcal{B}$ a suitable boundary operator associated with the $p$-Laplacian made more precise in Section 2. For most of the paper $f$ is a function only depending on $x$, but in Section [6 we consider problems with $f$ depending on $u$ as well. In particular we prove that in certain situations, every weak solution is in $L_{\infty}(\Omega)$. Section 5 is concerned with the existence and compactness of the resolvent operator associated with problem (1.1) in $L_{r}$-spaces. Problems similar to the above, often with $f=0$, were considered in $[10,12,13,19,20]$. Related local estimates for parabolic equations appear in 18 .

If $p=2$ (the linear case) it is well known that the solution $u$ is in $W_{r}^{2}(\Omega)$ if $f \in L_{r}(\Omega)$ and the domain $\Omega$ is sufficiently smooth. Also, $u$ satisfies an a priori estimate $\|u\|_{W_{r}^{2}} \leq c\left(\|f\|_{r}+\|u\|_{r}\right)$ with $c>0$ a constant independent of $f \in L_{r}(\Omega)$ due to [2]. By using embedding theorems for Sobolev spaces we obtain the estimate

$$
\|u\|_{m(r)} \leq c\left(\|f\|_{r}+\|u\|_{r}\right)
$$

with $m(r)=N r(N-2 r)^{-1}$ if $1<r<N / 2$ and $m(r)=\infty$ if $r>N / 2$. As shown in [7, such an estimate remains valid for a larger class of operators and non-smooth

Received by the editors November 2, 2007.

2000 Mathematics Subject Classification. Primary 35B45, 35B65, 35J65, 35J70.

Key words and phrases. Quasi-linear problems, $p$-Laplacian, $L_{p}$-estimates, non-smooth domains, Moser iterations.

The second author was supported by Research Plan MSM4977751301 of the Czech Ministry of Education, Youths and Sports. 
domains, even if the $W_{r}^{2}$-estimates fail. In the linear case, the exponent $m(r)$ is optimal and easy to guess from the embedding theorems for Sobolev spaces as mentioned above.

In this paper we want to generalise these estimates valid for the linear case $p=2$ to arbitrary $p \in(1, \infty)$. There are various difficulties involved with doing so. First, there is no good duality theory for (1.1) if $p \neq 2$. This is an important point, since in case $p=2$ the estimate (1.2) for $r \in(1,2)$ is obtained by using duality. This means that in certain cases we can only get estimates for $r \geq p^{\prime}$, where $p^{\prime}$ is the conjugate exponent to $p$ determined by $1 / p+1 / p^{\prime}=1$. Second, it is not so easy to guess the right exponent $m(r)$ if $p \neq 2$. One reason for this fact is that there are no $W_{r}^{2}$-estimates if $p \neq 2$; that is, if $f \in L_{r}(\Omega)$ we cannot expect that $u \in W_{r}^{2}(\Omega)$ even if $\Omega$ is smooth. We demonstrate this point by looking at the Dirichlet problem on an interval. Suppose now that $p>2$. It is well known that there exists a principal eigenvalue $\lambda_{1}$ and a principal eigenfunction $\varphi>0$ to the problem

$$
\begin{aligned}
-\left(\left|\varphi^{\prime}\right|^{p-2} \varphi^{\prime}\right)^{\prime} & =\lambda_{1}|\varphi|^{p-2} \varphi \quad \text { in }(0,1), \\
\varphi(0) & =\varphi(1)=0 .
\end{aligned}
$$

Also $\varphi \in C^{1}([0,1]), \varphi^{\prime}(1 / 2)=0$ (see [3]), and it follows from [8, Theorem 10.4] that $\left|\varphi^{\prime}\right|^{p-2} \varphi^{\prime} \in C^{1}([0,1])$. Integrating the first equation in (1.3) over $(0, x)$ we conclude that

$$
\varphi^{\prime}(x) \sim\left(x-\frac{1}{2}\right)^{1 /(p-1)} \text { and } \varphi^{\prime \prime}(x) \sim\left(x-\frac{1}{2}\right)^{-(p-2) /(p-1)}
$$

as $x \rightarrow 1 / 2$. Hence $\varphi \notin W_{r}^{2}(0,1)$ for $r>(p-1) /(p-2)$.

We next summarise our results. We seek weak solutions in a subspace $V_{p}$ of $W_{p}^{1}(\Omega)$ which incorporates the boundary conditions. For example $V_{p}=\stackrel{\circ}{W}_{p}^{1}(\Omega)$ in the case of homogeneous Dirichlet boundary conditions and $V_{p}=W_{p}^{1}(\Omega)$ in the case of homogeneous Neumann boundary conditions. We assume that $f \in L_{r}(\Omega) \cap V_{p}^{\prime}$, where $V_{p}^{\prime}$ is the dual of $V_{p}$, and that $V_{p} \hookrightarrow L_{d p /(d-p)}(\Omega)$ for some $d>p$ with embedding constant $c_{\mathcal{B}}$. The constant $c_{\mathcal{B}}$ depends on the boundary conditions and the domain in some cases. For precise conditions and examples see Assumption 2.4 and Section 4

The estimates for the solution $u$ of (1.1) we prove involve $c_{\mathcal{B}}$ and a constant $C>0$ depending only on $r$ and $1<p<d$. They have the form

$$
\|u\|_{\frac{d r(p-1)}{d-p r}}^{p-1} \leq C c_{\mathcal{B}} G(f, u)
$$

if $d p^{\prime} /\left(d+p^{\prime}\right) \leq r<d / p$ and

$$
\|u\|_{\infty}^{p-1} \leq \begin{cases}C c_{\mathcal{B}}|\Omega|^{\frac{p}{d}-\frac{1}{r}} G(f, u) & \text { if }|\Omega|<\infty \\ C c_{\mathcal{B}} G(f, u)+\|u\|_{p}^{p-1} & \text { if }|\Omega| \leq \infty\end{cases}
$$

if $r>d / p$. We do not have an estimate if $r=d / p$. There are two major cases to consider. The nicest case is the one of a coercive problem, where

$$
G(f, u)=\|f\|_{r}
$$

for all values of $r \geq d p^{\prime} /\left(d+p^{\prime}\right), r \neq d / p$ (see Theorem 2.5 for details). By coercive we mean that the form associated with the problem is positive definite; see Section 2, for a precise definition.

In the case of a non-coercive problem we can allow the same range of $r$ if $\Omega$ has finite measure, but if the measure is infinite we need to assume that $r \geq p^{\prime}$. Also, 
which form $G(f, u)$ takes depends on whether $r<p^{\prime}$ or $r \geq p^{\prime}$ (see Theorems 2.7 and 2.8 for details). We summarise the results in Table 1. The constant $\lambda_{0} \geq 0$ appearing there depends on the boundary conditions and $c_{0}$ and measures how far off the problem is from being coercive. (Note that for $r<p^{\prime} / p$ the term $\|u\|_{r(p-1)}$ is not actually a norm, but for convenience we use the same notation and definition as for $r \geq p^{\prime} / p$.) We discuss details in Section 2 and examples in Section 4. Since $r(p-1)<p$ if $r<p^{\prime}$ and so $L_{p}(\Omega) \not \subset L_{r(p-1)}(\Omega)$ if $|\Omega|=\infty$, our method does not provide an estimate if $|\Omega|=\infty$ and $r<p^{\prime}$ unless $p=2$. For $p=2$ an estimate can be obtained by duality (see [7]), but in general we do not know whether or not there is an estimate.

TABLE 1. Non-coercive problems, $\mu:=\frac{d}{p}\left(\frac{1}{p^{\prime}}-\frac{1}{r}\right)$.

\begin{tabular}{|c|c|c|c|}
\hline$r$ & $|\Omega|$ & $G(f, u)$ & $G(f, u) \quad$ (alternative) \\
\hline \hline$r<p^{\prime}$ & $|\Omega|<\infty$ & $\|f\|_{r}+\lambda_{0}|\Omega|^{\frac{1}{r}-\frac{1}{p^{\prime}}}\|u\|_{p}^{p-1}$ & $\|f\|_{r}+\lambda_{0}\|u\|_{r(p-1)}^{p-1}$ \\
\hline$r<p^{\prime}$ & $|\Omega|=\infty$ & no estimate for $p \neq 2$ & no estimate for $p \neq 2$ \\
\hline$r \geq p^{\prime}$ & $|\Omega| \leq \infty$ & $\|f\|_{r}+c_{\mathcal{B}}^{\mu} \lambda_{0}^{1+\mu}\|u\|_{p}^{p-1}$ & $\|f\|_{r}+\lambda_{0}\|u\|_{r(p-1)}^{p-1}$ \\
\hline
\end{tabular}

The basic method to prove our result originates from the seminal paper [16. We use suitable cutoff functions of the solution as test functions, interpolation inequalities and then do an iteration based on the validity of a Sobolev-type inequality for functions in $V_{p}$. This allows us to deal with arbitrary domains in the case of Dirichlet boundary conditions. It also allows "almost" arbitrary domains in the case of Robin-type boundary conditions by using an inequality due to Maz'ja [14, 15] and certain classes of non-smooth domains for Neumann boundary conditions (see Section 4). The proof of the main results is given in Section 3 .

\section{ASSUmptions AND MAIN RESUlts}

In this section we state our precise assumptions and then discuss our main results. We always assume that $\Omega$ is an open set. This set is not necessarily bounded or connected. The boundary $\partial \Omega$ is assumed to be the disjoint union of $\Gamma_{1}, \Gamma_{2}$ and $\Gamma_{3}$. We study regularity properties of the weak solutions of

$$
\left\{\begin{aligned}
-\Delta_{p} u+c_{0}|u|^{p-2} u & =f & & \text { in } \Omega, \\
u & =0 & & \text { on } \Gamma_{1} \text { (Dirichlet b.c.) } \\
|\nabla u|^{p-2} \frac{\partial u}{\partial \nu} & =0 & & \text { on } \Gamma_{2} \text { (Neumann b.c.) } \\
|\nabla u|^{p-2} \frac{\partial u}{\partial \nu}+b_{0}|u|^{p-2} u & =0 & & \text { on } \Gamma_{3} \text { (Robin b.c.). }
\end{aligned}\right.
$$


We assume that $c_{0} \in L_{\infty}(\Omega), b_{0} \in L_{\infty}\left(\Gamma_{3}\right), b_{0} \geq 0$ and that $\nu$ is the outward pointing unit normal to $\partial \Omega$. The reason we distinguish between $\Gamma_{2}$ and $\Gamma_{3}$ is that the assumptions we can make on the regularity of the boundary are different for $b_{0}=0$ and $b_{0}$ strictly positive. We refer to Section 4 for specific examples. The boundary conditions are to be understood in a weak sense as explained below. To define weak solutions of (2.1) we let

$$
a_{0}(u, v):=\int_{\Omega}|\nabla u|^{p-2} \nabla u \cdot \nabla v d x+\int_{\Omega} c_{0}(x)|u|^{p-2} u v d x
$$

for all $u, v \in W_{p}^{1}(\Omega)$. Then we define

$$
a(u, v):=a_{0}(u, v)+\int_{\Gamma_{3}} b_{0}|u|^{p-2} u v d \mathcal{H}_{N-1}
$$

whenever the last integral is well defined. Here, $\mathcal{H}_{N-1}$ denotes the $(N-1)$ dimensional Hausdorff measure, which coincides with the usual surface measure if $\Gamma_{3}$ is Lipschitz. We next give conditions on the space of test functions $V_{p}$ for the above problem.

Assumption 2.1. We assume that $V_{p}$ is a Banach space (with respect to a norm not necessarily equivalent to $\left.\|\cdot\|_{W_{p}^{1}}\right)$ such that

$$
\stackrel{\circ}{W}_{p}^{1}(\Omega) \hookrightarrow V_{p} \hookrightarrow W_{p}^{1}(\Omega),
$$

that

$$
\left\{u \in C_{c}^{1}\left(\bar{\Omega} \backslash \Gamma_{1}\right) \cap W_{p}^{1}(\Omega): \int_{\Gamma_{3}}|u|^{p} d \mathcal{H}_{N-1}<\infty\right\} \subset V_{p},
$$

and that the norm

$$
\|u\|_{V_{p}}:=\left(\|\nabla u\|_{p}^{p}+\|u\|_{p}^{p}+\left\|u \sqrt[p]{b_{0}}\right\|_{L_{p}\left(\Gamma_{3}\right)}^{p}\right)^{1 / p}
$$

is equivalent to the original norm on $V_{p}$. Finally we assume that

$$
u \min \left\{|u|^{t-1}, \alpha^{t-1}\right\} \in V_{p}
$$

for all $\alpha>0, t \geq 1$ and $u \in V_{p}$.

Remark 2.2. (a) Note that, depending on $b_{0}$ and $\Gamma_{3}$, the norm $\|\cdot\|_{V_{p}}$ may be stronger than the usual $W_{p}^{1}$-norm as shown in Section 4

(b) Since the $L_{p}$-norms are uniformly convex, the $V_{p}$-norm defined by (2.6) is also uniformly convex. Hence by Milman's theorem $V_{p}$ is a reflexive space (see [21, Section 5.2]).

Definition 2.3. Let $f \in V_{p}^{\prime}$. Then we call $u \in V_{p}$ a weak solution of (2.1) if $a(u, v)=\langle f, v\rangle$ for all $v \in V_{p}$.

In the above definition $\langle\cdot, \cdot\rangle$ denotes duality on $V_{p}$. Note that if $f \in L_{q}(\Omega)$ for some $q \in[1, \infty]$, then

$$
\langle f, v\rangle=\int_{\Omega} f v d x
$$

whenever the integral exists. We next introduce the main assumption implying our results on higher regularity. 
Assumption 2.4. Suppose that there exist $\delta_{0} \geq 0, c_{\mathcal{B}}>0$ and $d>p$ such that

$$
\|u\|_{d p /(d-p)}^{p} \leq c_{\mathcal{B}}\left(a(u, u)+\delta_{0}\|u\|_{p}^{p}\right)
$$

for all $u \in V_{p}$.

By the usual Sobolev embedding theorem the smallest possible constant is $d=N$, but it may well be that some $d>N$ is optimal. In particular this is the case for Neumann boundary conditions if the domain is not Lipschitz (see Section 4). We set

$$
\lambda_{0}:=\left\|c_{0}^{-}\right\|_{\infty}+\delta_{0} .
$$

If $\lambda_{0}=0$ we call the problem coercive, since then the functional $a(u, u)$ defined by (2.3) is coercive on $V_{p}$. If $\lambda_{0}>0$ we call the problem non-coercive.

Note also that the definition of a weak solution only makes sense if $f \in V_{p}^{\prime}$. By (2.7) we have $V_{p} \hookrightarrow L_{d p /(d-p)}(\Omega)$. Also, by Assumption $2.1 C_{c}^{\infty}(\Omega) \subset V_{p}$, and so $V_{p}$ is dense in $L_{p}(\Omega) \cap L_{d p /(d-p)}(\Omega)$. Hence by duality

$$
L_{\left(\frac{d p}{d-p}\right)^{\prime}}(\Omega) \hookrightarrow V_{p}^{\prime} .
$$

(Recall that $p^{\prime}$ is the conjugate exponent to $p$ given by $1 / p+1 / p^{\prime}=1$.) Note that

$$
L_{r}(\Omega) \hookrightarrow L_{\left(\frac{d p}{d-p}\right)^{\prime}}(\Omega)
$$

if $|\Omega|<\infty$ and

$$
r \geq\left(\frac{d p}{d-p}\right)^{\prime}=\frac{d p^{\prime}}{d+p^{\prime}} .
$$

Hence we consider (2.1) for $f \in L_{r}(\Omega)$ with $r$ as above. In the case of non-coercive problems we may need to assume that $r \geq p^{\prime}$ which obviously implies (2.9). Next we state the main results of the paper. We start with the easiest case, namely the case of a coercive problem.

Theorem 2.5 (Coercive problems). Suppose that Assumptions 2.1 and 2.4 hold with $\lambda_{0}=0$. Moreover, let $f \in L_{r}(\Omega) \cap V_{p}^{\prime}$ and let $u \in V_{p}$ be a weak solution of (2.11). Then there exists a constant $C>0$ depending only on $p, d$ and $r$ such that

$$
\|u\|_{\frac{d r(p-1)}{d-p r}}^{p-1} \leq C c_{\mathcal{B}}\|f\|_{r}
$$

if $d p^{\prime} /\left(d+p^{\prime}\right) \leq r<d / p$,

$$
\|u\|_{\infty}^{p-1} \leq C c_{\mathcal{B}}|\Omega|^{\frac{p}{d}-\frac{1}{r}}\|f\|_{r}
$$

if $r>d / p$ and $|\Omega|<\infty$, and

$$
\|u\|_{\infty}^{p-1} \leq C c_{\mathcal{B}}\|f\|_{r}+\|u\|_{p}^{p-1}
$$

if $r>d / p$ and there is no restriction on $|\Omega|$.

In the above theorem we have put various conditions on $d, p, r$, and we would like to check whether there are any non-obvious limitations.

Remark 2.6. It is not absolutely clear without further investigation whether the above theorem always gives additional regularity to solutions. Recall that we work 
with weak solutions, that is, the only thing we know initially is that $u \in V_{p}$. Due to the assumptions $V_{p} \hookrightarrow L_{d p /(d-p)}(\Omega)$, so naturally $u \in L_{d p /(d-p)}(\Omega)$. We set

$$
m(r):= \begin{cases}\frac{d r(p-1)}{d-p r} & \text { if } \frac{d p^{\prime}}{d+p^{\prime}} \leq r<\frac{d}{p}, \\ \infty & \text { if } r>\frac{d}{p} .\end{cases}
$$

An elementary calculation shows that

$$
m(r)>\frac{d p}{d-p} \quad \text { if and only if } \quad r>\frac{d p^{\prime}}{d+p^{\prime}} .
$$

Then, if $r>d p^{\prime} /\left(d+p^{\prime}\right)$ and $1<p<d$ (the assumption in the above theorem), then every weak solution of (2.1) with $f \in L_{r}(\Omega)$ is smoother than what the embedding theorem $V_{p} \hookrightarrow L_{d p /(d-p)}(\Omega)$ provides. Hence in any case, the above theorem gives additional information on the regularity of weak solutions to (2.1).

A second question we can ask is whether the solution has more regularity than the right hand side of the equation. More precisely, do we always have that $m(r)>r$ if $r>d p^{\prime} /\left(d+p^{\prime}\right)$ ? A simple calculation reveals that $m(r)>r$ if and only if

$$
r>\frac{d}{p}(2-p) \text {. }
$$

The right hand side is negative for $p \geq 2$, so this condition is always satisfied if $p \geq 2$. We have to be more careful if $1<p<2$. Then it depends on $d$ whether or not $m(r)>r$. An elementary calculation shows that if $d>2$, then

$$
\frac{d p^{\prime}}{d+p^{\prime}} \geq \frac{d}{p}(2-p) \quad \text { if and only if } \quad p \geq \frac{2 d}{d+2} .
$$

Hence, if $d>2$, then $m(r)>r$ for all $r>d p^{\prime} /\left(d+p^{\prime}\right)$ if and only if $p \geq 2 d /(d+2)$.

Next we state results valid for domains of finite measure, but possibly noncoercive problems.

Theorem 2.7 (Finite measure). Suppose that $|\Omega|<\infty$ and that Assumptions 2.1 and 2.4 hold. Moreover, let $f \in L_{r}(\Omega), r \geq d p^{\prime} /\left(d+p^{\prime}\right)$, and let $u \in V_{p}$ be a weak solution of (2.1). Then there exists a constant $C>0$ depending only on $p, d$ and $r$ such that

$$
\|u\|_{\frac{d r(p-1)}{d-p r}}^{p-1} \leq C c_{\mathcal{B}} G(f, u)
$$

if $d p^{\prime} /\left(d+p^{\prime}\right) \leq r<d / p$ and

$$
\|u\|_{\infty}^{p-1} \leq C c_{\mathcal{B}}|\Omega|^{\frac{p}{d}-\frac{1}{r}} G(f, u)
$$

if $r>d / p$, where either

$$
G(f, u)=\|f\|_{r}+\lambda_{0}\|u\|_{r(p-1)}^{p-1}
$$

or

$$
G(f, u)= \begin{cases}\|f\|_{r}+\lambda_{0}|\Omega|^{\frac{1}{r}-\frac{1}{p^{r}}}\|u\|_{p}^{p-1} & \text { if } r<p^{\prime}, \\ \|f\|_{r}+c_{\mathcal{B}}^{\mu} \lambda_{0}^{1+\mu}\|u\|_{p}^{p-1} & \text { if } r \geq p^{\prime},\end{cases}
$$

with $\mu:=\frac{d}{p}\left(\frac{1}{p^{\prime}}-\frac{1}{r}\right)$. 
Note that $r(p-1)<1$ if $r<p^{\prime} / p$, so $\|\cdot\|_{r(p-1)}$ is not a norm because it does not satisfy the triangle inequality. However, we simply understand it to be the integral $\left(\int_{\Omega}|u|^{r(p-1)} d x\right)^{1 / r(p-1)}$.

We finally give a result with no restriction on the measure of $\Omega$.

Theorem 2.8 (Arbitrary measure). Suppose that Assumptions 2.1 and 2.4 hold. Moreover, let $f \in L_{r}(\Omega) \cap V_{p}^{\prime}, r \geq p^{\prime}$, and let $u \in V_{p}$ be a weak solution of (2.1). Then there exists a constant $C>0$ depending only on $p, d$ and $r$ such that

$$
\|u\|_{\frac{d r(p-1)}{d-p r}}^{p-1} \leq C c_{\mathcal{B}} G(f, u)
$$

if $p^{\prime} \leq r<d / p$ and

$$
\|u\|_{\infty}^{p-1} \leq C c_{\mathcal{B}} G(f, u)+\|u\|_{p}^{p-1}
$$

if $r>d / p$ and $r \geq p^{\prime}$, where either

$$
G(f, u)=\|f\|_{r}+\lambda_{0}\|u\|_{r(p-1)}^{p-1}
$$

or

$$
G(f, u)=\|f\|_{r}+c_{\mathcal{B}}^{\mu} \lambda_{0}^{1+\mu}\|u\|_{p}^{p-1},
$$

with $\mu:=\frac{d}{p}\left(\frac{1}{p^{\prime}}-\frac{1}{r}\right)$.

\section{Proof of the Main Results}

The proof of the a priori estimates works by iteration. The iteration procedure is based on a basic inequality which we derive first. We assume that $V_{p}$ satisfies Assumptions 2.1 and 2.4. To make sure all relevant norms involved stay finite, we need to truncate the solution $u$ of (2.1). For $\alpha>0$ and $t \geq 1$ we set

$$
\psi_{\alpha, t}(\xi):=\xi \min \left\{\alpha^{t-1},|\xi|^{t-1}\right\} .
$$

Then we set

$$
v_{\alpha, q}:=\psi_{\alpha, q-p+1} \circ u \quad \text { and } \quad w_{\alpha, q}:=\psi_{\alpha, q / p} \circ u
$$

if $q \geq p$. It follows from [11, Theorem 7.8]) that $v_{\alpha, q}, w_{\alpha, q} \in W_{p}^{1}(\Omega)$ for all $\alpha>0$ and $q \geq p$ if $u \in W_{p}^{1}(\Omega)$. We also need to assume that $v_{\alpha, q}, w_{\alpha, q} \in V_{p}$ if $u \in V_{p}$, which is the case in all standard situations as shown in Section 4

Proposition 3.1. Suppose that Assumptions 2.1 and 2.4 hold. Moreover let $u$ be a weak solution of (2.1) with $f \in L_{r}(\Omega) \cap V_{p}^{\prime}$ for some $r \geq d p^{\prime} /\left(d+p^{\prime}\right)$. If $q \geq p$, then

$$
\left\|w_{\alpha, q}\right\|_{d p /(d-p)}^{p} \leq c_{\mathcal{B}} c(p, q)\left(\|f\|_{r}\|u\|_{r^{\prime}(q-p+1)}^{q-p+1}+\lambda_{0}\left\|w_{\alpha, q}\right\|_{p}^{p}\right)
$$

for all $\alpha>0$, where

$$
c(p, q):=\left(\frac{q}{p}\right)^{p} \frac{1}{q-p+1} .
$$

Proof. By Assumption 2.1 we have $v_{\alpha, q}, w_{\alpha, q} \in V_{p}$. It follows from [11, Theorem 7.8] that

$$
\nabla\left(\psi_{\alpha, t} \circ u\right)= \begin{cases}t|u|^{t-1} \nabla u & \text { if }|u|<\alpha \\ 0 & \text { if }|u|=\alpha \\ \alpha^{t-1} \nabla u & \text { if }|u|>\alpha\end{cases}
$$


for all $\alpha>0$ and $t \geq 1$. By the above we have

$$
\begin{aligned}
\left|\nabla w_{\alpha, q}\right|^{p} & =\left|\nabla\left(u|u|^{q / p-1}\right)\right|^{p}=\left(\frac{q}{p}\right)^{p}|u|^{q-p}|\nabla u|^{p} \\
& =\left(\frac{q}{p}\right)^{p} \frac{1}{q-p+1}|\nabla u|^{p-2} \nabla u \cdot \nabla\left(u|u|^{q-p}\right) \\
& =c(p, q)|\nabla u|^{p-2} \nabla u \cdot \nabla v_{\alpha, q}
\end{aligned}
$$

whenever $|u|<\alpha$, where $c(p, q)$ is defined by (3.3). Note that $c(p, q) \geq 1$ for all $q \geq p$. If $|u| \geq \alpha$, then

$$
\begin{aligned}
\left|\nabla w_{\alpha, q}\right|^{p}=|\nabla u|^{p} \alpha^{p(q / p-1)}= & |\nabla u|^{p-2} \nabla u \cdot \nabla\left(\alpha^{q-p} u\right) \\
& =|\nabla u|^{p-2} \nabla u \cdot \nabla v_{\alpha, q} \leq c(p, q)|\nabla u|^{p-2} \nabla u \cdot \nabla v_{\alpha, q}
\end{aligned}
$$

for all $q \geq p$ and $\alpha>0$. Combining the inequalities we get

$$
\left|\nabla w_{\alpha, q}\right|^{p} \leq c(p, q)|\nabla u|^{p-2} \nabla u \cdot \nabla v_{\alpha, q}
$$

for all $q \geq p$ and $\alpha>0$. Also, if $|u| \leq \alpha$, then

$$
\left|w_{\alpha, q}\right|^{p}=|u|^{q}=|u|^{p-2}|u|^{q-p+2}=|u|^{p-2} u^{2}|u|^{q-p}=|u|^{p-2} u v_{\alpha, q} .
$$

If $|u| \geq \alpha$, then

$$
\left|w_{\alpha, q}\right|^{p}=\alpha^{p(q / p-1)}|u|^{p}=|u|^{p-2} u^{2} \alpha^{q-p}=|u|^{p-2} u v_{\alpha, q} .
$$

Hence

$$
\left|w_{\alpha, q}\right|^{p}=|u|^{p-2} u v_{\alpha, q}
$$

for all $\alpha>0$ and $q \geq p$. If we note that $c(p, q) \geq 1$ and that $c_{0}(x)+\left\|c_{0}^{-}\right\|_{\infty} \geq 0$, then together with (3.5), (3.6) and (2.2)

$$
\begin{aligned}
a_{0}\left(w_{\alpha, q}, w_{\alpha, q}\right) \leq & c(p, q) \int_{\Omega}|\nabla u|^{p-2} \nabla u \cdot \nabla v_{\alpha, q} d x \\
+ & \int_{\Omega}\left(c_{0}(x)+\left\|c_{0}^{-}\right\|_{\infty}\right)\left|w_{\alpha, q}\right|^{p} d x \\
\leq & c(p, q)\left(\int_{\Omega}|\nabla u|^{p-2} \nabla u \cdot \nabla v_{\alpha, q} d x\right. \\
& \left.\quad+\int_{\Omega}\left(c_{0}(x)+\left\|c_{0}^{-}\right\|_{\infty}\right)\left|w_{\alpha, q}\right|^{p} d x\right) \\
= & c(p, q)\left(a_{0}\left(u, v_{\alpha, q}\right)+\left\|c_{0}^{-}\right\|_{\infty}\left\|w_{\alpha, q}\right\|_{p}^{p}\right) .
\end{aligned}
$$

Using the definition of $a$ given in (2.3) and the fact that $c(p, q) \geq 1$,

$$
a\left(w_{\alpha, q}, w_{\alpha, q}\right) \leq c(p, q)\left(a\left(u, v_{\alpha, q}\right)+\left\|c_{0}^{-}\right\|_{\infty}\left\|w_{\alpha, q}\right\|_{p}^{p}\right) .
$$

Since $w_{\alpha, q}, v_{\alpha, q} \in V_{p}$ it finally follows from Assumption 2.4 that

$$
\begin{aligned}
\left\|w_{\alpha, q}\right\|_{d p /(d-p)}^{p} \leq c_{\mathcal{B}}\left(a\left(w_{\alpha, q}, w_{\alpha, q}\right)+\right. & \left.\delta_{0}\left\|w_{\alpha, q}\right\|^{p}\right) \\
& \leq c_{\mathcal{B}} c(p, q)\left(a\left(u, v_{\alpha, q}\right)+\lambda_{0}\left\|w_{\alpha, q}\right\|_{p}^{p}\right)
\end{aligned}
$$

for all $\alpha>0$ and $q \geq p$. We next estimate the terms on the right hand side. First, since $u$ is a weak solution of (2.1) and $f \in L_{r}(\Omega)$ we get from Hölder's inequality

$$
a\left(u, v_{\alpha, q}\right)=\left\langle f, v_{\alpha, q}\right\rangle \leq\|f\|_{r}\left\|v_{\alpha, q}\right\|_{r^{\prime}} .
$$


By definition of $v_{\alpha, q}$ we have $\left|v_{\alpha, q}\right| \leq|u|^{q-p+1}$, so

$$
a\left(u, v_{\alpha, q}\right) \leq\|f\|_{r}\|u\|_{r^{\prime}(q-p+1)}^{q-p+1},
$$

and thus (3.2) follows if we combine everything.

Corollary 3.2. Suppose that Assumptions 2.1 and 2.4 hold. Moreover let $u$ be a weak solution of (2.1) with $f \in L_{r}(\Omega) \cap V_{p}^{\prime}$ for some $r \geq d p^{\prime} /\left(d+p^{\prime}\right)$. If $q \geq p$, then

$$
\left\|w_{\alpha, q}\right\|_{d p /(d-p)}^{p} \leq c_{\mathcal{B}} c(p, q)\left(\|f\|_{r}+\lambda_{0}\|u\|_{r(p-1)}^{p-1}\right)\|u\|_{r^{\prime}(q-p+1)}^{q-p+1}
$$

for all $\alpha>0$, where $c(p, q)$ is defined by (3.3).

Proof. By definition of $w_{\alpha, q}$ and Hölder's inequality,

$$
\left\|w_{\alpha, q}\right\|_{p}^{p} \leq\|u\|_{q}^{q}=\int_{\Omega}|u|^{q-p+1}|u|^{p-1} d x \leq\|u\|_{r(p-1)}^{p-1}\|u\|_{r^{\prime}(q-p+1)}^{q-p+1} .
$$

Substituting into (3.2), the assertion of the corollary follows.

Remark 3.3. If $\Omega$ has infinite measure we cannot expect $u \in L_{s}(\Omega)$ for $s \in[1, p)$. Hence in order for the right hand side of (3.8) to be finite we need that $r(p-1) \geq p$, that is, $r \geq p^{\prime}$ if $\lambda_{0} \neq 0$. Since $d p^{\prime} /\left(d+p^{\prime}\right) \leq p^{\prime}$ for all $1<p<d$ we need to assume that $r \geq p^{\prime}$ if we do not want our estimates to depend on the measure of $\Omega$. In the case of finite measure, $\|u\|_{s} \leq|\Omega|^{\frac{1}{s}-\frac{1}{p}}\|u\|_{p}<\infty$ even if we admit $s \in(0,1)$. In the latter case, $\|u\|_{s}:=\left(\int_{\Omega}|u|^{s} d x\right)^{1 / s}$ is not a norm, but for convenience we still use the same notation for the integral. In the coercive case where $\lambda_{0}=0$ the term involving $\|u\|_{r(p-1)}$ does not appear at all.

We next derive versions of the above inequality replacing $\|u\|_{r(p-1)}$ by $\|u\|_{p}$. According to the above remark we need to distinguish two cases, namely the case of $\Omega$ having finite measure or not.

Corollary 3.4 (Finite measure). Suppose that Assumptions 2.1 and 2.4 hold. Moreover let $u$ be a weak solution of (2.1) with $f \in L_{r}(\Omega)$ for some $d p^{\prime} /\left(d+p^{\prime}\right) \leq$ $r \leq p^{\prime}$. If $q \geq p$ and $|\Omega|<\infty$, then

$$
\left\|w_{\alpha, q}\right\|_{d p /(d-p)}^{p} \leq c_{\mathcal{B}} c(p, q)\left(\|f\|_{r}+\lambda_{0}|\Omega|^{1 / r-1 / p^{\prime}}\|u\|_{p}^{p-1}\right)\|u\|_{r^{\prime}(q-p+1)}^{q-p+1}
$$

for all $\alpha>0$, where $c(p, q)$ is defined by (3.3).

Proof. Since $|\Omega|<\infty$ and $r(p-1) \leq p$ we can apply Hölder's inequality with $s:=p^{\prime} / r \geq 1$ to get

$$
\|u\|_{r(p-1)}^{p-1}=\left(\int_{\Omega}|u|^{r(p-1)} d x\right)^{1 / r} \leq|\Omega|^{1 / r s^{\prime}}\|u\|_{p}^{p / r s}=|\Omega|^{1 / r-1 / p^{\prime}}\|u\|_{p}^{p-1} .
$$

Now the desired inequality follows from (3.8).

We finally establish a version for arbitrary measure. We need an interpolation inequality for that. It is similar to the standard one such as found in [11, page 146], but we admit $\|u\|_{s}:=\left(\int_{\Omega}|u|^{s} d s\right)^{1 / s}$ also for $s \in(0,1)$. We include the precise statement and proof for completeness. 
Lemma 3.5. Suppose that $0<s<p<t$ with $p \geq 1$. Set

$$
\mu:=\frac{t(p-s)}{s(t-p)} .
$$

Then

$$
\|u\|_{p}^{p} \leq \varepsilon^{-\mu}\|u\|_{s}^{p}+\varepsilon\|u\|_{t}^{p}
$$

for all $\varepsilon>0$ whenever the right hand side is finite.

Proof. We want to choose $\tau \in(0,1)$ and $\sigma, \rho \geq 1$ with $1 / p=1 / \sigma+1 / \rho$ such that $\tau \sigma=s$ and $(1-\tau) \rho=t$, and thus by Hölder's inequality

$$
\|u\|_{p}=\left\||u|^{\tau}|u|^{1-\tau}\right\|_{p} \leq\left\||u|^{\tau}\right\|_{\sigma}\left\||u|^{1-\tau}\right\|_{\rho}=\|u\|_{s}^{\tau}\|u\|_{t}^{1-\tau} .
$$

Solving the system of three equations for $\sigma, \rho$ and $\tau$ we get

$$
\sigma=p \frac{t-s}{t-p} \geq 1, \quad \rho=p \frac{t-s}{p-s} \geq 1, \quad \tau=\frac{s(t-p)}{p(t-s)} \in(0,1),
$$

so the above works. Next apply Young's inequality

$$
\begin{aligned}
\|u\|_{p} \leq\|u\|_{s}^{\tau}\|u\|_{t}^{1-\tau}=\left(\varepsilon^{-(1-\tau) / p \tau}\|u\|_{s}\right)^{\tau} & \left(\varepsilon^{1 / p}\|u\|_{t}\right)^{1-\tau} \\
& \leq \tau \varepsilon^{-(1-\tau) / p \tau}\|u\|_{s}+(1-\tau) \varepsilon^{1 / p}\|u\|_{t} .
\end{aligned}
$$

Finally, note that for $a, b \geq 0$ we have $a \leq\left(a^{p}+b^{p}\right)^{1 / p}$ and $b \leq\left(a^{p}+b^{p}\right)^{1 / p}$, so $\tau a+(1-\tau) b \leq\left(a^{p}+b^{p}\right)^{1 / p}$, and finally

$$
(\tau a+(1-\tau) b)^{p} \leq a^{p}+b^{p} .
$$

Hence from the above $\|u\|_{p}^{p} \leq \varepsilon^{-(1-\tau) / \tau}\|u\|_{s}^{p}+\varepsilon\|u\|_{t}^{p}$. Using the value for $\tau$ we get

$$
\mu=\frac{1-\tau}{\tau}=\frac{t(p-s)}{s(t-p)}
$$

as claimed.

Corollary 3.6 (Arbitrary measure). Suppose that Assumptions 2.1 and 2.4 hold. Moreover let $u$ be a weak solution of (2.1) with $f \in L_{r}(\Omega) \cap V_{p}^{\prime}$ for some $r \geq p^{\prime}$. If $q \geq p$, then there exists $c(q)>0$ depending on $r, d$ and $p$ such that

$$
\left\|w_{\alpha, q}\right\|_{d p /(d-p)}^{p} \leq c_{\mathcal{B}} c(q)\left(\|f\|_{r}+c_{\mathcal{B}}^{\mu} \lambda_{0}^{1+\mu}\|u\|_{p}^{p-1}\right)\|u\|_{r^{\prime}(q-p+1)}^{q-p+1}
$$

for all $\alpha>0$, where

$$
\mu:=\frac{d}{p}\left(\frac{1}{p^{\prime}}-\frac{1}{r}\right) \geq 0
$$

and the function $c(q) \geq 1$ grows at most polynomially in $q \geq p$.

Proof. We start from (3.2) and use an interpolation argument to absorb part of $\left\|w_{\alpha, q}\right\|_{p}^{p}$ on the left hand side. We first note that if $r \geq p^{\prime}$ and $d>p$, then

$$
\frac{p p^{\prime} r^{\prime}}{p^{\prime}+r^{\prime}} \leq p \leq \frac{d p}{d-p}
$$

Hence by the interpolation inequality from Lemma 3.5.

$$
\left\|w_{\alpha, q}\right\|_{p}^{p} \leq \varepsilon^{-\mu}\left\|w_{\alpha, q}\right\|_{p p^{\prime} r^{\prime} /\left(p^{\prime}+r^{\prime}\right)}^{p}+\varepsilon\left\|w_{\alpha, q}\right\|_{d p /(d-p)}^{p}
$$


with $\mu \geq 0$ given by (3.11). Now recall that $\left|w_{\alpha, q}\right|^{p} \leq|u|^{q}$. Hence by Hölder's inequality we have

$$
\begin{aligned}
\left\|w_{\alpha, q}\right\|_{p p^{\prime} r^{\prime} /\left(p^{\prime}+r^{\prime}\right)}^{p} \leq\left\|\left.u\right|^{p-1}|u|^{q-p+1}\right\|_{p^{\prime} r^{\prime} /\left(p^{\prime}+r^{\prime}\right)} & \\
& \leq\left\||u|^{p-1}\right\|_{p^{\prime}}\left\||u|^{q-p+1}\right\|_{r^{\prime}}=\|u\|_{p}^{p-1}\|u\|_{r^{\prime}(q-p+1)}^{q-p+1},
\end{aligned}
$$

and therefore

$$
\left\|w_{\alpha, q}\right\|_{p}^{p} \leq \varepsilon\left\|w_{\alpha, q}\right\|_{d p /(d-p)}^{p}+\varepsilon^{-\mu}\|u\|_{p}^{p-1}\|u\|_{r^{\prime}(q-p+1)}^{q-p+1}
$$

for all $\varepsilon>0$ and $q \geq p$. By (3.8)

$$
\begin{aligned}
\left\|w_{\alpha, q}\right\|_{d p /(d-p)}^{p} \leq c_{\mathcal{B}} c(p, q)\left(\|f\|_{r}+\lambda_{0} \varepsilon^{-\mu}\|u\|_{p}^{p-1}\right) & \|u\|_{r^{\prime}(q-p+1)}^{q-p+1} \\
& +\varepsilon c_{\mathcal{B}} c(p, q) \lambda_{0}\left\|w_{\alpha, q}\right\|_{d p /(d-p)}^{p} .
\end{aligned}
$$

Setting $\varepsilon:=\left(2 c_{\mathcal{B}} c(p, q) \lambda_{0}\right)^{-1}$ and moving the last term to the left hand side we get

$$
\left\|w_{\alpha, q}\right\|_{d p /(d-p)}^{p} \leq(2 c(p, q))^{1+\mu} c_{\mathcal{B}}\left(\|f\|_{r}+c_{\mathcal{B}}^{\mu} \lambda_{0}^{1+\mu}\|u\|_{p}^{p-1}\right)\|u\|_{r^{\prime}(q-p+1)}^{q-p+1}
$$

for all $q \geq p$. We also used the fact that $c(p, q) \geq 1$, and so $c(p, q) \leq c(p, q)^{1+\mu}$. If we set $c(q):=(2 c(p, q))^{1+\mu}$ the assertion of the corollary follows.

The inequalities derived above all have the form

$$
\left\|w_{\alpha, q}\right\|_{d p /(d-p)}^{p} \leq c(q) c_{\mathcal{B}} G(f, u)\|u\|_{r^{\prime}(q-p+1)}^{q-p+1}
$$

for an appropriate function $G(f, u)$, where $c(q)$ grows at most polynomially in $q \geq p$. In particular we have the following cases:

(1) If $\lambda_{0}=0$ and $r \geq d p^{\prime} /\left(d+p^{\prime}\right)$, then

$$
G(f, u)=\|f\|_{r}
$$

and $c(q):=c(p, q)$ by Proposition 3.1 (coercive case).

(2) If $\lambda_{0} \geq 0$, and $r \geq d p^{\prime} /\left(d+p^{\prime}\right)$ if $|\Omega|<\infty$ and $r \geq p^{\prime}$ otherwise, then

$$
G(f, u)=\|f\|_{r}+\lambda_{0}\|u\|_{r(p-1)}^{p-1}
$$

and $c(q):=c(p, q)$ by Corollary 3.2 ,

(3) If $|\Omega|<\infty, \lambda_{0} \geq 0$ and $d p^{\prime} /\left(d+p^{\prime}\right) \leq r \leq p^{\prime}$, then

$$
G(f, u)=\|f\|_{r}+\lambda_{0}|\Omega|^{1 / r-1 / p^{\prime}}\|u\|_{p}^{p-1}
$$

and $c(q):=c(p, q)$ by Corollary 3.4 .

(4) If $\lambda_{0} \geq 0$ and $r \geq p^{\prime}$, then

$$
G(f, u)=\|f\|_{r}+c_{\mathcal{B}}^{\mu} \lambda_{0}^{1+\mu}\|u\|_{p}^{p-1}
$$

and $c(q):=(2 c(p, q))^{1+\mu}$ by Corollary 3.6, where $\mu$ is defined by (3.11).

We now implement an iteration procedure based on (3.12) which allows us to prove all versions of a priori estimates stated if we take into account the above. As $\left|w_{\alpha, q}\right|^{p} \nearrow|u|^{q}$ as $\alpha \rightarrow \infty$ it follows from (3.12) and the monotone convergence theorem that

$$
\|u\|_{d q /(d-p)}^{q} \leq c(q) c_{\mathcal{B}} G(f, u)\|u\|_{r^{\prime}(q-p+1)}^{q-p+1}
$$


whenever the right hand side is finite. Assuming that $G(f, u)$ is finite and non-zero, we set

$$
v:=\frac{u}{\left(c_{\mathcal{B}} G(f, u)\right)^{1 /(p-1)}}
$$

Then (3.17) turns into

$$
\|v\|_{d q /(d-p)}^{q} \leq c(q)\|v\|_{r^{\prime}(q-p+1)}^{q-p+1} .
$$

The idea then is to iterate the inequality by choosing an initial $q_{0}$ and computing $q_{n+1}$ from $q_{n}$ by solving the equation

$$
\frac{d q_{n}}{d-p}=r^{\prime}\left(q_{n+1}-p+1\right)
$$

It turns out that

$$
q_{n+1}=\eta q_{n}+p-1
$$

with

$$
\eta:=\frac{d}{r^{\prime}(d-p)}
$$

If we do that (3.19) turns into

$$
\|v\|_{d q_{n+1} /(d-p)}^{q_{n+1}} \leq c\left(q_{n+1}\right)\|v\|_{d q_{n} /(d-p)}^{\eta q_{n}}
$$

for all $n \in \mathbb{N}$. The right hand side is certainly finite for $n=0$ by (2.7) if we set $q_{0}=$ $p$. The above inequality tells us that $u \in L_{d q_{1} /(d-p)}(\Omega)$. Applying the inequality again we conclude that $u \in L_{d q_{2} /(d-p)}(\Omega)$ and iterating $n$ times $u \in L_{d q_{n} /(d-p)}(\Omega)$ for all $n \in \mathbb{N}$. However, for this to be useful we need to know that $\left(q_{n}\right)$ is an increasing sequence. By the recursion formula (3.20) and induction we have

$$
q_{n}=\eta^{n} q_{0}+(p-1) \sum_{k=0}^{n-1} \eta^{k}=\eta^{n}\left(q_{0}-p+1\right)+(p-1) \sum_{k=0}^{n} \eta^{k},
$$

and therefore

$$
q_{n}=\eta^{n}\left(q_{0}-p+1\right)+(p-1) \sum_{k=0}^{n} \eta^{k} .
$$

We now prove that $\left(q_{n}\right)$ is increasing if we set $q_{0}:=p$. Then from (3.23)

$$
q_{n+1}-q_{n}=\eta^{n+1}-\eta^{n}+(p-1) \eta^{n+1}=\eta^{n}(\eta p-1)
$$

for all $n \in \mathbb{N}$. The above is positive if and only if $\eta p-1>0$ which is the case if and only if

$$
\frac{d p}{d-p}>r^{\prime}
$$

if we use the definition of $\eta$. The above is equivalent to the assumption (2.9) on $r$, so we really have an improvement of regularity at each iteration step. If $r=d p^{\prime} /\left(d+p^{\prime}\right)$, then $q_{n}=p$ for all $n \in \mathbb{N}$ as expected in Remark 2.6. Our a priori estimates are a consequence of the following lemma. 
Lemma 3.7. Suppose that (3.22) holds, $v$ is defined by (3.18), and $q_{0} \geq p$ is such that $u \in L_{d q_{0} /(d-p)}(\Omega)$. Then

$$
\|v\|_{d q_{n} /(d-p)}^{q_{n}} \leq\left(\prod_{k=1}^{n} c\left(q_{k}\right)^{\eta^{n-k}}\right)\|v\|_{d q_{0} /(d-p)}^{\eta^{n} q_{0}}
$$

for all $n \in \mathbb{N}$.

Proof. We give a proof by induction. For $n=1$, (3.24) reduces to (3.22) for $n=0$. If $n>1$, then by (3.22) and the induction assumption

$$
\begin{aligned}
\|v\|_{d q_{n+1} /(d-p)}^{q_{n+1}} & \leq c\left(q_{n+1}\right)\|v\|_{d q_{n} /(d-p)}^{\eta q_{n}} \\
& \leq c\left(q_{n+1}\right)\left(\prod_{k=1}^{n} c\left(q_{k}\right)^{\eta^{n-k}}\right)^{\eta}\|v\|_{d q_{0} /(d-p)}^{\eta^{n+1} q_{0}} \\
& =c\left(q_{n+1}\right)\left(\prod_{k=1}^{n} c\left(q_{k}\right)^{\eta^{n-k+1}}\right)\|v\|_{d q_{0} /(d-p)}^{\eta^{n+1} q_{0}} \\
& =\left(\prod_{k=1}^{n+1} c\left(q_{k}\right)^{\eta^{(n+1)-k}}\right)\|v\|_{d q_{0} /(d-p)}^{\eta^{n+1} q_{0}},
\end{aligned}
$$

which is exactly what we need.

Theorem 3.8. Suppose that (3.12) holds, that $d p^{\prime} /\left(d+p^{\prime}\right) \leq r$ and that $q_{0} \geq p$. If $G(f, u)$ is finite and non-zero, then there exists a constant $C$ depending only on $d$, $p, r$ and the function $c(q)$ such that

$$
\|u\|_{\frac{d r(p-1)}{d-p r}}^{p-1} \leq C c_{\mathcal{B}} G(f, u)
$$

if $r<d / p$ and

$$
\|u\|_{\infty}^{q_{0}+\frac{r(d-p)(p-1)}{r p-d}} \leq C c_{\mathcal{B}} G(f, u)^{\frac{r(d-p)}{r p-d}}\|u\|_{d q_{0} /(d-p)}^{q_{0}}
$$

if $r>d / p$.

Proof. First consider the case $d p^{\prime} /\left(d+p^{\prime}\right) \leq r<d / p$. Let $\eta$ be as defined in (3.21) and set $q_{0}:=p$. Then $\eta \in(0,1)$, and therefore by (3.23)

$$
\bar{q}:=\lim _{n \rightarrow \infty} q_{n}=\frac{p-1}{1-\eta}=\frac{d r(p-1)}{d-p r} \frac{d-p}{d} .
$$

As $\left(q_{n}\right)$ is an increasing sequence and $c(q)$ monotone in $q \geq p$ we get from (3.24)

$$
\begin{aligned}
\|v\|_{d q_{n} /(d-p)}^{q_{n}} \leq c(\bar{q})^{\sum_{k=1}^{n} \eta^{n-k}}\|v\|_{d p /(d-p)}^{\eta^{n} p} & \\
& =c(\bar{q})^{\sum_{k=0}^{n-1} \eta^{k}}\|v\|_{d p /(d-p)}^{\eta^{n} p} \leq c(\bar{q})^{1 /(1-\eta)}\|v\|_{d p /(d-p)}^{\eta^{n} p} .
\end{aligned}
$$

As $\eta^{n} \rightarrow 0$ and $q_{n} \rightarrow \bar{q}$ it follows from Fatou's lemma that

$$
\|v\|_{\frac{d r(p-1)}{d-p r}}^{\bar{q}}=\|v\|_{d \bar{q} /(d-p)}^{\bar{q}} \leq \liminf _{n \rightarrow \infty}\|v\|_{d q_{n} /(d-p)}^{q_{n}} \leq c(\bar{q})^{1 /(1-\eta)} .
$$

Using the definition of $v$ the estimate (3.25) follows.

Assume now that $r>d / p$ and that $q_{0} \geq p$ is such that $u \in L_{d q_{0} /(d-p)}(\Omega)$. Using that $\eta>1$ we claim that

$$
\eta^{n} q_{0} \leq q_{n} \leq(2 \eta)^{n} q_{0}
$$


for all $n \in \mathbb{N}$. We give a proof by induction. For $n=0$ the inequality is obvious. Suppose now that (3.27) holds for some $n \geq 0$. Since $\eta>1$ and $p-1 \leq p \leq q_{0} \leq$ $\eta^{n+1} q_{0} \leq \eta q_{n}$ it follows that

$$
\eta^{n+1} q_{0} \leq \eta q_{n} \leq \eta q_{n}+p-1=q_{n+1} \leq 2 \eta q_{n} \leq(2 \eta)^{n+1} q_{0},
$$

as required. Hence (3.27) holds for all $n \geq 0$. If we take the $\eta^{n}$-th root of (3.24) we get

$$
\|v\|_{d q_{n} /(d-p)}^{q_{n} \eta^{-n}} \leq\left(\prod_{k=1}^{n} c\left(q_{k}\right)^{\eta^{-k}}\right)\|v\|_{d q_{0} /(d-p)}^{q_{0}}
$$

for all $n \geq 1$. Next we derive a bound for the product in the above inequality by using the fact that $c(q)$ has polynomial growth. By assumption there exist $\beta \geq 1$ and $t \geq 1$ such that

for all $q \geq p$. Using (3.27) we see that

$$
c(q) \leq \beta q^{t}
$$

$$
c\left(q_{k}\right) \leq \beta(2 \eta)^{k t} q_{0}^{t} \leq\left(2 \beta q_{0} \eta\right)^{k t}
$$

for all $k \geq 1$. Hence (3.28) implies that

$$
\begin{aligned}
&\|v\|_{d q_{n} /(d-p)}^{q_{n} \eta^{-n}} \leq\left(\prod_{k=1}^{n}\left(2 \beta q_{0} \eta\right)^{k t \eta^{-k}}\right)\|v\|_{d q_{0} /(d-p)}^{q_{0}} \\
& \leq\left(2 \beta q_{0} \eta\right)^{t \sum_{k=1}^{\infty} k \eta^{-k}}\|v\|_{d q_{0} /(d-p)}^{q_{0}},
\end{aligned}
$$

where the series in the exponent converges since $\eta>1$. Hence if we set

$$
C:=\left(2 \beta q_{0} \eta\right)^{t \sum_{k=1}^{\infty} k \eta^{-k}}<\infty
$$

we get

$$
\|v\|_{d q_{n} /(d-p)}^{q_{n} \eta^{-n}} \leq C\|v\|_{d q_{0} /(d-p)}^{q_{0}}
$$

for all $n \in \mathbb{N}$ with $C \geq 1$ independent of $n \in \mathbb{N}$. In order to let $n \rightarrow \infty$ we need to compute the limit of $q_{n} \eta^{-n}$. From (3.23), using the fact that $\eta>1$, we get

$$
\begin{aligned}
\lim _{n \rightarrow \infty} \frac{q_{n}}{\eta^{n}}=q_{0}-p+1+ & (p-1) \sum_{k=0}^{\infty} \eta^{-k}=q_{0}-p+1+\frac{p-1}{1-\eta^{-1}} \\
& =q_{0}-p+1+\frac{d(r-1)(p-1)}{r p-d}=q_{0}+\frac{r(d-p)(p-1)}{r p-d} .
\end{aligned}
$$

Letting $n \rightarrow \infty$ in (3.29) and noting that $q_{n} \rightarrow \infty$ we get

$$
\|v\|_{\infty}^{q_{0}+\frac{r(d-p)(p-1)}{r p-d}}=\lim _{n \rightarrow \infty}\|v\|_{d q_{n} /(d-p)}^{q_{n} \eta^{-n}} \leq C\|v\|_{d q_{0} /(d-p)}^{q_{0}},
$$

which is equivalent to (3.26) if we take into account (3.18).

Remark 3.9. If $r<d / p$ we could attempt to get (3.25) from (3.17) by calculating $q$ such that

$$
\frac{d q}{d-p}=r^{\prime}(q-p+1)
$$

Solving the equation for $q$ we get

$$
q=\frac{r^{\prime}(p-1)(d-p)}{r^{\prime}(d-p)-d}
$$


Note that $q>p$ since $r<d / p$. Therefore,

$$
\frac{d q}{d-p}=r^{\prime}(q-p+1)=\frac{d r(p-1)}{d-p r}
$$

and we get

$$
\|u\|_{\frac{d r(p-1)}{d-p r}}^{q} \leq c(q) c_{\mathcal{B}} G(f, u)\|u\|_{\frac{d r(p-1)}{d-p r}}^{q-p+1} .
$$

Now (3.25) follows by dividing the above inequality by $\|u\|_{\frac{d r(p-1)}{d-p r}}^{q-p+1}$. However, this approach does not work since we do not a priori know whether $u \in L_{\frac{d r(p-1)}{d-p r}}(\Omega)$. Hence we had to use an iteration argument to show that.

We next derive another estimate in the case $r>d / p$.

Corollary 3.10. Suppose the assumptions of the above theorem are satisfied and that $r>d / p$. If $u \in L_{\infty}(\Omega)$, then there exists a constant $C>0$ depending only on $d, p$ and $r$ such that

$$
\|u\|_{\infty}^{p-1} \leq C c_{\mathcal{B}}|\Omega|^{\frac{p}{d}-\frac{1}{r}} G(f, u)
$$

if $\Omega$ has finite measure and

$$
\|u\|_{\infty}^{p-1} \leq C c_{\mathcal{B}} G(f, u)+\|u\|_{p}^{p-1}
$$

or

$$
\|u\|_{\infty}^{p-1} \leq C c_{\mathcal{B}} G(f, u)+\|u\|_{r(p-1)}^{p-1}
$$

otherwise.

Proof. Let $v$ be defined by (3.18). Then $v \in L_{p}(\Omega) \cap L_{\infty}(\Omega)$ and thus $v \in$ $L_{d q_{0} /(d-p)}(\Omega)$ for all $q_{0} \geq p$. First suppose that $\Omega$ has finite measure. Then by Hölder's inequality

$$
\|v\|_{\frac{d p}{d-p}}^{p} \leq|\Omega|^{(d-p) / d}\|v\|_{\infty}^{p} .
$$

Substituting this into (3.30), setting $q_{0}=p$, we get

$$
\|v\|_{\infty}^{p+\frac{r(d-p)(p-1)}{r p-d}} \leq C|\Omega|^{(d-p) / d}\|v\|_{\infty}^{p} .
$$

Rearranging and using the definition of $v$ we get (3.31) by renaming the constant $C$. Let $s \geq p$ and choose $q_{0} \geq p$ such that $s \leq d q_{0} /(d-p)$. By interpolation we get

$$
\|v\|_{\frac{d q_{0}}{d-p}}^{q_{0_{0}}} \leq\|v\|_{s}^{s(1-p / d)}\|v\|_{\infty}^{q_{0}-s(1-p / d)} .
$$

If we set

$$
\delta:=\frac{d r(p-1)}{r p-d}
$$

then (3.30) implies

$$
\|v\|_{\infty}^{q_{0}+\delta(1-p / d)} \leq C\|v\|_{s}^{s(1-p / d)}\|v\|_{\infty}^{q_{0}-s(1-p / d)} .
$$

Dividing by $\|v\|_{\infty}^{q_{0}-s(1-p / d)}$ we have

$$
\|v\|_{\infty}^{(\delta+s)(1-p / d)} \leq C\|v\|_{s}^{s(1-p / d)}
$$

or equivalently

$$
\|v\|_{\infty}^{(\delta+s)} \leq C^{d /(d-p)}\|v\|_{s}^{s} .
$$


Using the definition of $\delta$ and Young's inequality

$$
\|v\|_{\infty}^{p-1} \leq C^{\frac{r p-d}{r(d-p)} \frac{\delta}{s+\delta}}\|v\|_{s}^{(p-1) \frac{s}{s+\delta}} \leq \frac{\delta}{s+\delta} C^{\frac{r p-d}{r(d-p)}}+\frac{s}{s+\delta}\|v\|_{s}^{p-1} .
$$

Renaming the constant $C$ we get

$$
\|v\|_{\infty}^{p-1} \leq C+\|v\|_{s}^{p-1} .
$$

Now if we choose $s:=p$ we get (3.32), and if $r \geq p^{\prime}$ and if we choose $s:=r(p-1)$ we get (3.33) by using the definition of $v$, completing the proof of the corollary.

We now derive the main theorems stated in Section 2 ,

Proof of Theorem 2.5. Suppose that the problem is coercive; that is, $\lambda_{0}=0$. As seen in (3.13) we can set $G(f, u):=\|f\|_{r}$. Hence if $d p^{\prime} /(d-p) \leq r<d / p$, then by Theorem 3.8 the estimate (2.10) follows immediately. Now let $r>d / p$. If we set $\gamma:=r(d-p) /(r p-d)$ and $q_{0}:=p$, we get from Theorem 3.8 that

$$
\|u\|_{\infty}^{p+\gamma(p-1)} \leq C\left(c_{\mathcal{B}}\|f\|_{r}\right)^{p+\gamma}\|u\|_{d p /(d-p)}^{p}
$$

where the right hand side is finite because $u \in L_{d p /(d-p)}(\Omega)$ by (2.7). Hence $u \in L_{\infty}(\Omega)$. Now (2.11) and (2.12) follow from Corollary 3.10, completing the proof of Theorem 2.5,

Proof of Theorem 2.7. Suppose that $|\Omega|<\infty$ and that $\lambda_{0} \geq 0$. If $r<p^{\prime}$ we let $G(f, u)$ be as in (3.15), and if $r \geq p^{\prime}$ we let $G(f, u)$ be as in (3.16). In both cases $G(f, u)<\infty$ since $u \in L_{p}(\Omega)$ and $f \in L_{r}(\Omega)$. Now if $d p^{\prime} /\left(d+p^{\prime}\right) \leq r<p / d$, then (2.14) for the above choice of $G(f, u)$ follows from (3.25).

Now let $r>d / p$. Then we see that $u \in L_{\infty}(\Omega)$ by setting $q_{0}=p$ in (3.26). Hence (2.15) follows from Corollary 3.10.

In each of the above cases we get the fact that $u \in L_{r(p-1)}(\Omega)$ since $r(p-1) \leq$ $d r(p-1) /(d-p)$. Hence we can use $G(f, u)$ as defined in (3.14) instead of (3.15), proving the remaining assertion of Theorem 2.7

Proof of Theorem [2.8. If $|\Omega|$ is possibly infinite and $\lambda_{0} \geq 0$, then the proof is similar to the one for Theorem 2.7 but we can only apply the arguments for $r \geq p^{\prime}$ (see also Remark 3.31).

\section{EXAMPles AND REMARKS}

In this section we discuss some examples where our results apply. We essentially look at the Dirichlet, Neumann and Robin problems separately and identify the spaces $V_{p}$ and the "dimension" $d$ appearing in the embedding inequality (2.7). We present only some model problems, but many kinds of mixed problems are also possible. We give a general criterion for the last part of Assumption 2.1 in Appendix A. We also prove there that it applies to the standard examples discussed below.

Dirichlet boundary conditions. We assume that $\partial \Omega=\Gamma_{1}$ and let $V_{p}:=\stackrel{\circ}{W}_{p}^{1}(\Omega)$, which is by definition the closure of the set of test functions having compact support in the arbitrary open set $\Omega \subset \mathbb{R}^{N}$ in $W_{p}^{1}(\Omega)$. It is well known that if $N>p$, then there exists a constant $c$ depending only on $N$ and $p$ such that

$$
\|u\|_{N p /(N-p)} \leq c\|\nabla u\|_{p}
$$

for all $u \in \stackrel{\circ}{W}_{p}^{1}(\Omega)$ (see [11, Theorem 7.10]). We can therefore set $d:=N$ in (2.7) and $\delta_{0}:=0$ in Assumption 2.4. Hence if $c_{0} \geq 0$, then $\lambda_{0}=0$, so the problem is 
coercive for any open set. If $|\Omega|<\infty$ we can replace $N$ by any $d \geq N$ and find a constant also depending on $|\Omega|$ such that

$$
\|u\|_{d p /(d-p)} \leq c\|\nabla u\|_{p}
$$

for all $u \in \stackrel{\circ}{W}_{p}^{1}(\Omega)$. In particular, given $p>1$ we can choose $d \geq N$ such that $p<d$ and then apply our results. But of course the estimates become weaker the larger we choose $d$.

Neumann boundary conditions. We assume that $\Omega$ is a bounded Lipschitz domain and that $\partial \Omega=\Gamma_{2}$ or $\Gamma_{3}$ with $b_{0} \geq 0$ in (2.1). We can choose $V_{p}:=W_{p}^{1}(\Omega)$. It is well known that if $N>p$, then there exists a constant $c$ depending on $N, p$ and the domain such that the Sobolev inequality

$$
\|u\|_{N p /(N-p)} \leq c\|u\|_{W_{p}^{1}}
$$

holds for all $u \in W_{p}^{1}(\Omega)$ (see [17, Théorème 3.4]). We can therefore set $d:=N$ in (2.7) and $\delta_{0}:=1$ in Assumption 2.4 Hence $\lambda_{0}=1+\left\|c_{0}^{-}\right\|_{\infty}>0$, so the problem is not in general coercive. If $\gamma:=\min c_{0}>0$, then

$$
\|u\|_{W_{p}^{1}}^{p} \leq \max \left\{1, \gamma^{-1}\right\}\left(\|\nabla u\|_{p}^{p}+\gamma\|u\|_{p}^{p}\right) \leq a(u, u)
$$

and therefore we can set $\lambda_{0}=0$ if $\min c_{0}>0$, meaning that the problem is coercive in that case. We can replace $N$ by any $d \geq N$ and find a constant also depending on $|\Omega|$ such that

$$
\|u\|_{d p /(d-p)} \leq c\|u\|_{W_{p}^{1}}
$$

for all $u \in W_{p}^{1}(\Omega)$. If $\Omega$ is not Lipschitz and $\partial \Omega=\Gamma_{2}$, then (2.7) can fail for any $d \geq N$. An example is a domain with an outward pointing exponential cusp as shown in [1, Theorem 5.32]. On the other hand, there are domains for which (2.7) holds for some optimal $d>N$. Model examples are again domains with outward pointing polynomial cusps (see [1, Theorem 5.35]). If $p>d$ we can simply increase $d$ since for a domain with finite measure, (2.7) holds for any $d$ larger than the minimal possible $d$ and then apply our results.

Robin boundary conditions on (almost) arbitrary domains. Now we suppose that $\Omega$ is an arbitrary domain and $\partial \Omega=\Gamma_{3}$ and that there exists a constant $\beta>0$ such that $b_{0} \geq \beta$. We then set

$$
V_{p}:=W_{p, p}^{1}(\Omega, \partial \Omega),
$$

which is defined to be the completion of the space

$$
\left\{u \in W_{p}^{1}(\Omega) \cap C(\bar{\Omega}):\|u\|_{V_{p}}<\infty\right\}
$$

with respect to the norm

$$
\|u\|_{V_{p}}=\left(\|u\|_{W_{p}^{1}}^{p}+\left\|\left.u\right|_{\partial \Omega}\right\|_{L_{p}(\partial \Omega)}^{p}\right)^{1 / p},
$$

where $L_{p}(\partial \Omega)$ is given with respect to the $(N-1)$-dimensional Hausdorff measure. These spaces have been introduced by Maz'ja (see [15, Section 3.6]). We prove in the Appendix that Assumption 2.1 is satisfied. Moreover, if $N>1$, by [15, Corollary 3.6.3] there exists a constant $c>0$ just depending on $N$ (namely the isoperimetric constant) such that

$$
\|u\|_{N /(N-1)} \leq c\left(\|\nabla u\|_{1}+\left\|\left.u\right|_{\partial \Omega}\right\|_{L_{1}(\partial \Omega)}\right)
$$


for all $u \in W_{1}^{1}(\Omega) \cap C(\bar{\Omega})$ for which the right hand side is finite. Replacing $u$ by $|u|^{p}$ we get

$$
\|u\|_{N p /(N-1)}^{p} \leq c\left(p\left\|\left.|| u\right|^{p-1}\left|\nabla u\left\|_{1}+\right\| u\right|_{\partial \Omega}\right\|_{L_{p}(\partial \Omega)}^{p}\right) .
$$

By Hölder's and Young's inequalities

$$
p\left\||u|^{p-1}|\nabla u|\right\|_{1} \leq p\|u\|_{p}^{p-1}\|\nabla u\|_{p} \leq(p-1)\|u\|_{p}^{p}+\|\nabla u\|_{p}^{p},
$$

and hence there exists $C>0$ only depending on $N$ and $p$ such that

$$
\|u\|_{N p /(N-1)} \leq C\|u\|_{V_{p}}
$$

for all $u \in V_{p}$. Now clearly

$$
\frac{N p}{N-1}=\frac{(N p) p}{N p-p}
$$

so if we set $d:=N p$, then (2.7) is satisfied. Unfortunately, there are domains for which the embedding $V_{p} \rightarrow L_{p}(\Omega)$ is not injective, since $V_{p}$ is only defined as an abstract completion of a normed space (see [5] for an example in the case $p=2$ ). Hence we assume that the embedding is injective and call a domain with that property admissible. For such domains we get a priori estimates of the type discussed in this paper if we set $d=N p$. Note that $d>p$ for all $N>1$. If the domain has finite measure and $c_{0} \geq 0$, then the problem turns out to be coercive. Using Hölder's and Young's inequality we have

$$
c p\left\||u|^{p-1}|\nabla u|\right\|_{1} \leq c p|\Omega|^{\frac{1}{N p^{\prime}}}\|u\|_{N p /(N-1)}^{p-1}\|\nabla u\|_{p} \leq c_{1}\|u\|_{N p /(N-1)}^{p}+\frac{1}{p}\|\nabla u\|_{p}^{p}
$$

for some constant $c_{1}$ only depending on $N, p$ and $|\Omega|$. Rearranging (4.1) we get a constant $C>0$ such that

$$
\|u\|_{N p /(N-1)} \leq C\left(\|\nabla u\|_{p}^{p}+\left\|\left.u\right|_{\partial \Omega}\right\|_{L_{p}(\partial \Omega)}^{p}\right)^{1 / p} .
$$

Clearly

$$
\int_{\partial \Omega} b_{0}|u|^{p} d \sigma \geq \beta \int_{\partial \Omega}|u|^{p} d \sigma=\beta\left\|\left.u\right|_{\partial \Omega}\right\|_{L_{p}(\partial \Omega)}^{p}
$$

and hence

$$
\|u\|_{N p /(N-1)}^{p} \leq C^{p} \max \left\{1, \beta^{-1}\right\} a(u, u) .
$$

We can therefore set $d=N p$ and $\delta_{0}=0$ under the above assumptions. This means that the problem is coercive for every admissible bounded domain if $c_{0} \geq 0$.

Finally note that for instance for a domain with an outward pointing exponential cusp, $\|\cdot\|_{V_{p}}$ is stronger than the $W_{p}^{1}$-norm, and thus by the open mapping theorem the space $V_{p}$ is a proper subspace of $W_{p}^{1}(\Omega)$. That the norm is strictly stronger follows from [1, Theorem 5.32], asserting that for a domain with a sufficiently sharp outward pointing cusp $W_{p}^{1}(\Omega) \not \subset L_{q}(\Omega)$ for all $q>p$, contradicting (4.2) if we assume that $V_{p}=W_{p}^{1}(\Omega)$.

Note also that one may be able to deal with arbitrary open sets $\Omega$ by replacing $V_{p}$ by an appropriate closed subspace as done in [6, Section 3] or [5] for the linear case. 


\section{Existence AND COMPACTNESS OF THE RESOlVEnt}

In this section we look at existence and compactness of the resolvent to the problem (2.1) under the Assumptions 2.1 and 2.4. We also assume that $\Omega$ is bounded, that the problem is coercive, and that the embedding

$$
V_{p} \hookrightarrow L_{p}(\Omega)
$$

is compact. (We write $\hookrightarrow$ for a compact embedding.) We prove that the solution operator $T^{-1}: L_{r}(\Omega) \rightarrow V_{p} \cap L_{m(r)}$ exists for all $r>d p^{\prime} /\left(d+p^{\prime}\right)$ and that it is compact as an operator into $V_{p} \cap L_{s}(\Omega)$ for all $s \in(1, m(r))$, where $m(r)$ is as defined in (2.13). Compact means that it maps bounded sets onto relatively compact sets. We start by constructing the solution operator. By our assumptions, there exists $c>0$ such that

$$
|a(u, v)| \leq c\|u\|_{V_{p}}^{p-1}\|v\|_{V_{p}}
$$

for all $u, v \in V_{p}$. In particular, this shows that for every fixed $u \in V_{p}$ the functional $v \mapsto a(u, v)$ is an element of the dual space $V_{p}^{\prime}$. Hence for each $u \in V_{p}$ there exists $T(u) \in V_{p}^{\prime}$ such that

$$
\langle T(u), v\rangle=a(u, v)
$$

for all $u, v \in V_{p}$ and therefore defines an operator $T: V_{p} \rightarrow V_{p}^{\prime}$. This map is continuous essentially because the superposition operator associated with the function $g(\xi):=|\xi|^{p-2} \xi$ is continuous from $L_{p}(\Omega)$ to $L_{p^{\prime}}(\Omega)$ and also from $L_{p}\left(\Gamma_{3}\right)$ to $L_{p^{\prime}}\left(\Gamma_{3}\right)$ (see [8, page 188]). The monotonicity of $g$ implies that $T$ is a monotone operator, and the assumption on the coercivity of the problem guarantees that $T$ is a coercive operator as well. We show that $T$ is bounded, that is, it maps bounded sets of $V_{p}$ onto bounded sets in $V_{p}^{\prime}$. By definition of the dual norm and (5.1)

$$
\|T(u)\|_{V_{p}^{\prime}}=\sup _{\|v\|_{V_{p}}=1}|\langle T(u), v\rangle|=\sup _{\|v\|_{V_{p}}=1}|a(u, v)| \leq c \sup _{\|v\|_{V_{p}}=1}\|u\|_{V_{p}}^{p-1}\|v\|_{V_{p}},
$$

and thus $T$ is bounded. It follows from the Browder theorem (see [9, Theorem 5.3.22]) that $T\left(V_{p}\right)=V_{p}^{\prime}$, that is, $T$ maps $V_{p}$ onto $V_{p}^{\prime}$. We next prove existence, continuity and boundedness of the operator $T^{-1}$, also between $L_{s}$-spaces.

Theorem 5.1. Suppose that Assumptions 2.1 and 2.4 hold and that $c_{0}, b_{0} \geq 0$. Then $T^{-1}: V_{p}^{\prime} \rightarrow V_{p}$ exists, is bounded and continuous. Moreover, if $r \geq \bar{d} p^{\prime} /$ $\left(d+p^{\prime}\right)$, then $T^{-1}: L_{r}(\Omega) \rightarrow L_{s}(\Omega)$ is bounded and continuous for all $s \in[1, m(r))$, where $m(r)$ is given by (2.13).

Proof. Since the map $\xi \rightarrow|\xi|^{p-2} \xi$ is strictly monotone, it follows that

$$
\left(|\xi|^{p-2} \xi-|\eta|^{p-2} \eta\right)(\xi-\eta)>0
$$

for all $\xi \neq \eta$. Hence, by the coercivity of the problem and the definition of $T$ and $a(u, v)$, it follows that

$$
\langle T(u)-T(v), u-v\rangle>0
$$

for all $u, v \in V_{p}$ with $u \neq v$. Note that the only possibility for the above expression to be zero is if $\nabla u=\nabla v=0$ almost everywhere and $c_{0}=b_{0}=0$. But then the problem is not coercive, contrary to what we assumed. Hence $T$ is injective and $T^{-1}$ exists. The coercivity also implies the existence of a constant $C>0$ such that

$$
C\|u\|_{V_{p}}^{p} \leq a(u, u)=\langle T(u), u\rangle
$$


for all $u \in V_{p}$. Hence if $u \in V_{p}$ is the weak solution of (2.1) with $f \in V_{p}^{\prime}$, then

$$
C\|u\|_{V_{p}}^{p} \leq\langle T(u), u\rangle=\langle f, u\rangle \leq\|f\|_{V_{p}^{\prime}}\|u\|_{V_{p}},
$$

and since $u=T^{-1} f$,

$$
\left\|T^{-1}(f)\right\|_{V_{p}}^{p-1} \leq C^{-1}\|f\|_{V_{p}^{\prime}}
$$

for all $f \in V_{p}^{\prime}$. Thus $T^{-1}$ is bounded on $V_{p}^{\prime}$. We next show that $T^{-1}$ is continuous on $V_{p}^{\prime}$. From Hölder's inequality and (5.2) we get

$$
\begin{aligned}
\langle T(u)- & T(v), u-v\rangle \\
\geq & \int_{\Omega}\left(|\nabla u|^{p-2} \nabla u-|\nabla v|^{p-2} \nabla v\right) \cdot(\nabla u-\nabla v) d x \\
= & \|\nabla u\|_{p}^{p}+\|\nabla v\|_{p}^{p} \\
& \quad \quad-\int_{\Omega}|\nabla u|^{p-2} \nabla u \cdot \nabla v d x-\int_{\Omega}|\nabla v|^{p-2} \nabla v \cdot \nabla u d x \\
\geq & \|\nabla u\|_{p}^{p}+\|\nabla v\|_{p}^{p}-\|\nabla u\|_{p}^{p-1}\|v\|_{p}-\|\nabla v\|_{p}^{p-1}\|u\|_{p} \\
= & \left(\|\nabla u\|_{p}^{p-1}-\|\nabla v\|_{p}^{p-1}\right)\left(\|\nabla u\|_{p}-\|\nabla v\|_{p}\right)
\end{aligned}
$$

for all $u, v \in V_{p}$. Similarly, for all $u, v \in V_{p}$,

$$
\langle T(u)-T(v), u-v\rangle \geq\left(\left\|c_{0}^{1 / p} u\right\|_{p}^{p-1}-\left\|c_{0}^{1 / p} v\right\|_{p}^{p-1}\right)\left(\left\|c_{0}^{1 / p} u\right\|_{p}-\left\|c_{0}^{1 / p} v\right\|_{p}\right),
$$

and if $\Gamma_{3} \neq \emptyset$,

$$
\begin{aligned}
\langle T(u)-T(v), u-v\rangle \geq\left(\left\|b_{0}^{1 / p} u\right\|_{L_{p}\left(\Gamma_{3}\right)}^{p-1}-\right. & \left.\left\|c_{b}^{1 / p} v\right\|_{L_{p}\left(\Gamma_{3}\right)}^{p-1}\right) \\
& \cdot\left(\left\|b_{0}^{1 / p} u\right\|_{L_{p}\left(\Gamma_{3}\right)}-\left\|b_{0}^{1 / p} v\right\|_{L_{p}\left(\Gamma_{3}\right)}\right) .
\end{aligned}
$$

Assume to the contrary that $T^{-1}$ is not continuous. Then there exist $f_{n} \in V_{p}^{\prime}$ with $f_{n} \rightarrow f$ in $V_{p}^{\prime}$ and $\delta>0$ such that

$$
\left\|T^{-1}\left(f_{n}\right)-T^{-1}(f)\right\|_{V_{p}} \geq \delta
$$

for all $n \in \mathbb{N}$. Set $u_{n}:=T^{-1}\left(f_{n}\right)$ and $u:=T^{-1}(f)$. As $\left(f_{n}\right)$ is a bounded sequence and $T^{-1}$ is bounded, the sequence $\left(u_{n}\right)$ is bounded in $V_{p}$. By the reflexivity of $V_{p}$ (see Remark 2.2) it has a weakly convergent subsequence, so by renumbering it we can assume that $\left(u_{n}\right)$ converges weakly to some $\tilde{u} \in V_{p}$. Since by assumption $T\left(u_{n}\right)-T(\tilde{u}) \rightarrow f-T(\tilde{u})$ strongly in $V_{p}^{\prime}$ and $u_{n}-\tilde{u} \rightarrow 0$ weakly in $V_{p}$, we get

$$
\left\langle T\left(u_{n}\right)-T(\tilde{u}), u_{n}-\tilde{u}\right\rangle \rightarrow 0
$$

as $n \rightarrow \infty$. Setting $u=u_{n}$ and $v=\tilde{u}$ in (5.3) (5.5) we conclude that

$$
\begin{aligned}
a\left(u_{n}, u_{n}\right)=\left\|\nabla u_{n}\right\|_{p}^{p}+\left\|c_{0}^{1 / p} u_{n}\right\|_{p}^{p}+\left\|b_{0}^{1 / p} u_{n}\right\|_{L_{p}\left(\Gamma_{3}\right)}^{p} & \\
& \rightarrow\|\nabla \tilde{u}\|_{p}^{p}+\left\|c_{0}^{1 / p} \tilde{u}\right\|_{p}^{p}+\left\|b_{0}^{1 / p} \tilde{u}\right\|_{L_{p}\left(\Gamma_{3}\right)}^{p}=a(\tilde{u}, \tilde{u}) .
\end{aligned}
$$

By the coercivity $a(u, u)^{1 / p}$ is a strictly convex equivalent norm on the uniformly convex Banach space $V_{p}$, showing that $u_{n} \rightarrow \tilde{u}$ strongly in $V_{p}$. Since $T$ is continuous, $f_{n}=T\left(u_{n}\right) \rightarrow T(\tilde{u})=f$, and by injectivity $\tilde{u}=u$. This means that

$$
\left\|T^{-1}\left(f_{n}\right)-T^{-1}(f)\right\|_{V_{p}}=\left\|u_{n}-u\right\|_{V_{p}} \rightarrow 0
$$

contradicting (5.6). Hence $T^{-1}$ is continuous on $V_{p}^{\prime}$, as claimed. To show that $T^{-1}: L_{r}(\Omega) \rightarrow L_{m(r)}(\Omega)$ is bounded let $B \subset L_{r}(\Omega)$ be bounded. From Theorem 2.5 
we know that $\left\|T^{-1}(f)\right\|_{m(r)} \leq C\|f\|_{r}$ for some constant $C$ independent of $f \in$ $L_{r}(\Omega)$. Hence the image of $B$ under $T^{-1}$ is bounded in $L_{m(r)}(\Omega)$.

We finally show that $T^{-1}: L_{r}(\Omega) \rightarrow L_{s}(\Omega)$ is continuous for all $s \in[1, m(r)$ ). Since $V_{p} \hookrightarrow L_{d p /(d-p)}(\Omega)$ it is sufficient to look at $s \geq d p /(d-p)$. Suppose now that $r \geq d p^{\prime} /\left(d+p^{\prime}\right)$ and let $\left(f_{n}\right)$ be a sequence in $L_{r}(\Omega)$ with $f_{n} \rightarrow f$ in $L_{r}(\Omega)$. Then as shown above, the sequence $T^{-1}\left(f_{n}\right)$ is bounded in $L_{m(r)}(\Omega)$. Also $T^{-1}: L_{r}(\Omega) \hookrightarrow$ $V_{p}^{\prime} \rightarrow V_{p} \hookrightarrow L_{d p /(d-p)}(\Omega)$ is continuous. Hence if $s \in[d p /(d-p), m(r))$, then by interpolation, for some $\tau \in(0,1]$,

$$
\begin{aligned}
& \left\|T^{-1}\left(f_{n}\right)-T^{-1}(f)\right\|_{s} \\
& \leq\left\|T^{-1}\left(f_{n}\right)-T^{-1}(f)\right\|_{d p /(d-p)}^{\tau}\left\|T^{-1}\left(f_{n}\right)-T^{-1}(f)\right\|_{m(r)}^{1-\tau} \\
& \quad \leq C\left\|T^{-1}\left(f_{n}\right)-T^{-1}(f)\right\|_{V_{p}}^{\tau} \rightarrow 0
\end{aligned}
$$

as $n \rightarrow \infty$ by continuity of $T^{-1}$ as a map into $V_{p}$. This concludes the proof of the theorem.

We next look at the compactness of $T^{-1}$.

Theorem 5.2. Suppose that Assumptions 2.1 and 2.4 hold and that $c_{0}, b_{0} \geq 0$. Further assume that $\Omega$ is bounded, that the problem is coercive, and that the embedding $V_{p} \hookrightarrow L_{p}(\Omega)$ is compact. Then the solution operator $T^{-1}: L_{r}(\Omega) \rightarrow V_{p} \cap L_{s}(\Omega)$ is compact for all $r>d p^{\prime} /\left(d+p^{\prime}\right)$ and $s \in(1, m(r))$.

Proof. By Theorem $5.1 T^{-1}: V_{p}^{\prime} \rightarrow V_{p}$ exists and is bounded and continuous. By assumption and interpolation we have

$$
V_{p} \hookrightarrow L_{t}(\Omega)
$$

for $1<t<d p /(d-p)$, and therefore by duality

$$
L_{r}(\Omega) \hookrightarrow V_{p}^{\prime}
$$

for all $r>d p^{\prime} /\left(d+p^{\prime}\right)$. Hence by continuity and boundedness of $T^{-1}: V_{p}^{\prime} \rightarrow V_{p}$ it follows that

$$
T^{-1}: L_{r}(\Omega) \rightarrow V_{p}
$$

is compact for $r>d p^{\prime} /\left(d+p^{\prime}\right)$. We now show that $T^{-1}$ is also compact as a map into $L_{s}$ for $s \in(1, m(r))$. From Theorem 5.1 we know that $T^{-1}$ is bounded. We need to show that the image of every bounded set $B \subset L_{r}(\Omega)$ is relatively compact in $L_{s}(\Omega)$ for $1<s<m(r)$. For that it is sufficient to show that every sequence in $T^{-1}(B)$ has a convergent subsequence in $L_{s}(\Omega)$. Hence let $\left(u_{n}\right)$ be a sequence in $T^{-1}(B)$. Let $f_{n}:=T\left(u_{n}\right) \in B$ and note that because $B$ is bounded, the sequence $\left(f_{n}\right)$ is bounded. We have already seen that $T^{-1}$ is a compact map into $V_{p}$, so there is a subsequence $\left(f_{n_{k}}\right)$ such that $T^{-1}\left(f_{n_{k}}\right) \rightarrow u$ in $V_{p}$. Renumbering the sequence we can assume that $u_{n}=T^{-1}\left(f_{n}\right) \rightarrow u$ in $V_{p}$ and therefore in $L_{p}(\Omega)$. It remains to show that $u_{n} \rightarrow u$ in $L_{s}(\Omega)$. Now let $s \in(p, m(r))$. Then, as $\left(u_{n}\right)$ is bounded in $L_{m(r)}(\Omega)$, a standard interpolation inequality (see also the proof of Lemma 3.5) implies that there exists $\tau \in(0,1)$ such that

$$
\left\|u_{n}-u_{m}\right\|_{s} \leq\left\|u_{n}-u_{m}\right\|_{p}^{\tau}\left\|u_{n}-u_{m}\right\|_{m(r)}^{1-\tau} \leq 2 C\left\|u_{n}-u_{m}\right\|_{p}^{\tau}
$$

for all $n, m \in \mathbb{N}$. As $\left(u_{n}\right)$ converges in $L_{p}(\Omega)$ we conclude that $\left(u_{n}\right)$ is a Cauchy sequence in $L_{s}(\Omega)$ and therefore converges in $L_{s}(\Omega)$. Hence $T^{-1}: L_{r}(\Omega) \rightarrow L_{s}(\Omega)$ is compact for $s \in[p, m(r))$. For $s \in(1, p)$ simply observe that $L_{p}(\Omega) \hookrightarrow L_{s}(\Omega)$ because $\Omega$ has finite measure. 
Remark 5.3. In general we do not expect $T^{-1}: L_{r}(\Omega) \rightarrow L_{m(r)}(\Omega)$ to be compact. The reason is that $V_{p} \hookrightarrow L_{d p /(d-p)}(\Omega)$ is not compact if $d \geq N$ is optimal. At least in the linear case there is a converse of the a priori estimates which would imply compactness of the embedding (see [7, Section 6]).

\section{Non-Linear SOURCES}

We now apply the results from Section 2 to problems with $f$ depending on $u$ as well. We consider

$$
\begin{aligned}
-\Delta_{p} u+c_{0}|u|^{p-2} u & =f(x, u(x)) & & \text { in } \Omega, \\
\mathcal{B} u & =0 & & \text { on } \partial \Omega .
\end{aligned}
$$

Throughout we work with the framework introduced in Section 2 and in particular rely on Assumptions 2.1 and 2.4. On $f$ we make the following assumptions.

Assumption 6.1. Suppose that $f: \Omega \times \mathbb{R} \rightarrow \mathbb{R}$ is a Carathéodory function, that is, $f(\cdot, \xi): \mathbb{R} \rightarrow \mathbb{R}$ is measurable for all $\xi \in \mathbb{R}$, and $f(x, \cdot) \in C(\mathbb{R})$ for almost all $x \in \Omega$. Further suppose that there exist a measurable function $g: \Omega \rightarrow[0, \infty)$ and constants $1 \leq \beta<\infty$ and $c \geq 0$ such that

$$
|f(x, \xi)| \leq g(x)+c|\xi|^{\beta}
$$

for all $(x, \xi) \in \Omega \times \mathbb{R}$.

We define the superposition operator $F$ acting on the measurable function $u: \Omega$ $\rightarrow \mathbb{R}$ by

$$
F(u)(x):=f(x, u(x))
$$

for all $x \in \Omega$. The following result follows from [4, Theorems 3.1 and 3.7].

Lemma 6.2. If $g \in L_{s / \beta}(\Omega)$, then $F \in C\left(L_{s}(\Omega), L_{s / \beta}(\Omega)\right)$ with

$$
\|F(u)\|_{s / \beta} \leq\|g\|_{s / \beta}+c\|u\|_{s}
$$

for all $u \in L_{s}(\Omega)$.

We call $u \in V_{p}$ a weak solution of (6.1) if $F(u) \in V_{p}^{\prime}$ and

$$
a(u, v)=\langle F(u), v\rangle
$$

for all $v \in V_{p}$. We look at a weak solution $u$ lying in $L_{s}(\Omega)$ for some $s \geq d p /(d-p)$ and show that it lies in an even better space. For the problem to make sense we need to know that $F(u) \in V_{p}^{\prime}$. To achieve that we impose restrictions on the growth $\beta$. Assuming that

$$
p-1 \leq \beta<p-1+\frac{p}{d} s
$$

and that $g \in V_{p}^{\prime}$, we prove that $F(u) \in V_{p}^{\prime}$.

Lemma 6.3. Suppose that Assumptions 2.1 and 2.4 hold. Moreover, let $F$ be as above with $\beta$ satisfying (6.4) and $g \in V_{p}^{\prime}$. Then $F(u) \in V_{p}^{\prime}$ for all $u \in V_{p} \cap L_{s}(\Omega)$.

Proof. Let $u_{1}(x):=u(x)$ if $|u(x)| \leq 1$ and $u_{1}(x):=0$ if $|u(x)|>1$. If we set $u_{2}:=u-u_{1}$, then clearly $\left|u_{2}(x)\right|>1$ whenever $u_{2}(x) \neq 0$. Given $v \in V_{p}$ we have

$$
\begin{aligned}
|\langle F(u), v\rangle| \leq\langle|F(u)|,|v|\rangle \leq\langle g,|v|\rangle & +c\left\langle|u|^{\beta},|v|\right\rangle \\
& \leq\|g\|_{V_{p}^{\prime}}\|v\|_{V_{p}}+c\left\langle\left|u_{1}\right|^{\beta},|v|\right\rangle+c\left\langle\left|u_{2}\right|^{\beta},|v|\right\rangle .
\end{aligned}
$$


Since $\beta \geq p-1$ we clearly have $\left|u_{1}\right|^{\beta} \leq\left|u_{1}\right|^{p-1}$. Moreover, by (6.4)

$$
m(s / \beta)>s \geq \frac{d p}{d-p}=m\left(\frac{d p^{\prime}}{d+p^{\prime}}\right) .
$$

Since $m$ is an increasing function

$$
\frac{s}{\beta}>\frac{d p^{\prime}}{d+p^{\prime}}
$$

and therefore $\left|u_{2}\right|^{\beta} \leq\left|u_{2}\right|^{s\left(d+p^{\prime}\right) / d p^{\prime}}$. Using Hölder's inequality we get

$$
\left\langle\left|u_{1}\right|^{\beta},|v|\right\rangle+\left\langle\left|u_{2}\right|^{\beta},|v|\right\rangle \leq\left\|u_{1}\right\|_{p}^{p-1}\|v\|_{p}+\left\|u_{2}\right\|_{s}^{s\left(d+p^{\prime}\right) / d p^{\prime}}\|v\|_{d p /(d-p)} .
$$

As $V_{p} \hookrightarrow L_{p}(\Omega) \cap L_{d p /(d-p)}(\Omega)$ there exists a constant $C>0$ such that

$$
|\langle F(u), v\rangle| \leq C\left(\|g\|_{V_{p}^{\prime}}+\left\|u_{1}\right\|_{p}+\left\|u_{2}\right\|_{s}\right)\|v\|_{V_{p}} .
$$

Hence $F(u) \in V^{\prime}$, as claimed.

We next state the main theorem of the section. Recall from (2.13) the definition of $m(r)$.

Theorem 6.4. Suppose that Assumptions 2.1 and 2.4 hold with $\lambda_{0}=0$ or $|\Omega|<\infty$. Let $u \in L_{s}(\Omega)$ be a weak solution of (6.1) for some $s \geq d p /(d-p)$ and $\beta$ a constant such that (6.4) holds. Finally, let $s / \beta<r \neq d / p$ and $g \in L_{r}(\Omega) \cap L_{s / \beta}(\Omega)$. If $f$ satisfies Assumption 6.1 with $g$ and $\beta$ as above, then $u \in L_{m(r)}(\Omega)$.

Before we give a proof of the theorem we discuss some special cases and possible modifications.

Corollary 6.5. Suppose that Assumptions 2.1 and 2.4 hold with $\lambda_{0}=0$ or $|\Omega|<$ $\infty$. Let $u$ be a weak solution of (6.1) and $\beta$ a constant such that

$$
p-1 \leq \beta<p-1+\frac{p^{2}}{d-p} .
$$

Moreover, let $s / \beta<r \neq d / p$ and $g \in L_{r}(\Omega) \cap L_{s / \beta}(\Omega)$. If $f$ satisfies Assumption 6.1 with the above $g$ and $\beta$, then $u \in L_{m(r)}(\Omega)$.

Proof. Note that (6.4) turns into (6.5) if we set $s=d p /(d-p)$. By assumption, every weak solution lies in $L_{d p /(d-p)}(\Omega)$, and so (6.4) is automatically satisfied and Theorem 6.4 applies.

Corollary 6.6. Suppose that $f$ is a Carathéodory function on $\Omega \times \mathbb{R}$ and $b, c \geq 0$ are constants such that

$$
|f(x, \xi)| \leq b+c|\xi|^{\beta}
$$

for all $(x, \xi) \in \Omega \times \mathbb{R}$. If $|\Omega|<\infty$ and (6.5) is satisfied, then every weak solution of (6.1) is in $L_{\infty}(\Omega)$.

Example 6.7. (a) Note that in the linear case $p=2$, condition (6.5) corresponds precisely to the case of sub-critical growth $1 \leq \beta<(d+2) /(d-2)$ of the nonlinearity.

(b) If $f(x, u)=g(x)+h(x, u)$ with $h \sim|u|^{p-2} u$, then (6.5) is automatically satisfied with $\beta=p-1$ and the theorem applies. This is even the case if $|\Omega|=\infty$ and $\lambda_{0}>0$ since automatically $s \geq d p /(d-p)>p$, and so $p-1<s / p^{\prime}$ as required in Remark 6.8(b) below. 
Remark 6.8. (a) As in Remark 2.6 we discuss under what circumstances Theorem 6.4 provides new information on the solution $u$ of (6.1). First we note that new information is obtained only if $d p /(d-p) \leq s<m(r)$, because otherwise $u \in L_{m(r)}(\Omega)$ already by interpolation. Next note that $m(r)>s$ if and only if

$$
r>\frac{s}{p-1+\frac{p}{d} s} .
$$

In Theorem 6.4 we assume that $r>s / \beta$. Suppose now that $r$ satisfies condition (6.6). If $|\Omega|<\infty$, we can replace $\beta$ by some suitable larger $\beta$ still satisfying (6.4) such that $r>s / \beta$. Hence the condition that $r>s / \beta$ is no restriction in that case. If $|\Omega|=\infty$ this is a minor additional condition on $r$.

(b) In Theorem 6.4 we assumed that $\lambda_{0}=0$ or $|\Omega|<\infty$. If neither is true we need to modify the assumptions. For the argument in the proof to be valid we need in addition that $r \geq p^{\prime}$ and that $\beta \leq s / p^{\prime}$. Then the same conclusion holds.

We conclude this section by a proof of Theorem 6.4.

Proof of Theorem 6.4. The idea of the proof is once again an iteration or bootstrapping argument. We define

$$
s_{0}:=s \quad \text { and } \quad s_{n+1}:=m\left(s_{n} / \beta\right) \text { for } n \geq 0 .
$$

Note that the strict inequality in (6.4) is equivalent to $s=s_{0}<m\left(s_{0} / \beta\right)=s_{1}$. Since $m(\cdot)$ is increasing, by induction the sequence $\left(s_{n}\right)$ is increasing as long as $s_{n} / \beta<d / p$. Note that $m(d / p)$ is not defined, so we have to avoid $s_{n} / \beta=d / p$ for some $n$. Since $u \in L_{p}(\Omega) \cap L_{s}(\Omega)$ we can do that by choosing $s$ slightly smaller, making sure (6.4) remains true. Now since $s<s_{n}$ and $d(p-1) /(d \beta-p s)>1$, by (6.4) we have

$$
s_{n+1}-s_{n}=\frac{d(p-1) s_{n}}{d \beta-p s_{n}}-s_{n}=\left(\frac{d(p-1)}{d \beta-p s_{n}}-1\right) s_{n} \geq\left(\frac{d(p-1)}{d \beta-p s}-1\right) s
$$

as long as $s_{n} / \beta<d / p$. From the above it is impossible that $s_{n} / \beta<d / p$ for all $n \in \mathbb{N}$, and hence there exists $n \in \mathbb{N}$ such that $s_{n} / \beta<d / p<s_{n+1} / \beta$. But then by the definition $s_{n+2}=m\left(s_{n+1} / \beta\right)=\infty$.

By the properties of $\left(s_{n}\right)$ we just proved, there exists $\ell \in \mathbb{N}$ such that

$$
s_{\ell}<m(r) \leq s_{\ell+1} .
$$

By induction we now show that $u \in L_{s_{n}}(\Omega)$ for $n=0, \ldots, \ell$. For $n=0$ this is true by assumption. Now suppose that $u \in L_{s_{n}}(\Omega)$ for some $n<\ell$. Since $n<\ell$ we have $s / \beta \leq s_{n} / \beta<r$. By the assumption on $g$ and interpolation, $g \in L_{s_{n} / \beta}(\Omega)$, and so by Lemma 6.2 we get $F(u) \in L_{s_{n} / \beta}(\Omega)$. Now Theorems 2.5 or 2.7 imply $u \in L_{s_{n+1}}(\Omega)$, as claimed. Also observe that $s_{1}<m(r)$ because $r>s / \beta$, and so $\ell \geq 1$. If $s_{\ell+1}<\infty$ it follows from (6.7), the definition of $\left(s_{n}\right)$ and the monotonicity of the function $m(\cdot)$ that $s \leq s_{\ell-1}<\beta r \leq s_{\ell}$. Moreover, $u \in L_{s}(\Omega) \cap L_{s_{\ell}}(\Omega)$, and so by interpolation $u \in L_{\beta r}(\Omega)$. Hence $F(u) \in L_{r}(\Omega)$ and Theorems 2.5 or 2.7 finally imply $u \in L_{m(r)}(\Omega)$, as claimed. If $s_{\ell+1}=\infty$, then there are two possibilities. First, as above, $s \leq s_{\ell-1}<\beta r \leq s_{\ell}$, and the claim follows in the same way. Second, we could have $s \leq s_{\ell-1}<s_{\ell}<\beta r$, and in that case by interpolation $g \in L_{s_{\ell} / \beta}(\Omega)$ and hence $F(u) \in L_{s_{\ell} / \beta}(\Omega)$ by Lemma 6.2. Finally Theorems 2.5 or 2.7 imply that $u \in L_{s_{\ell+1}}(\Omega)$, completing the proof of the theorem since $s_{\ell+1}=\infty$. 


\section{Appendix A. Invariance of $V_{p}$}

The purpose of this appendix is to establish a general criterion to show that $\psi_{\alpha, t} \circ u \in V_{p}$ for all $u \in V_{p}$ (the final requirement in Assumption 2.1) for a large class of problems, in particular for the boundary conditions considered in Section 4 . The criterion applies in a similar manner to more general mixed problems.

Proposition A.1. Suppose that $V_{p}$ is a Banach space such that (2.4), (2.5) holds, and that (2.6) is an equivalent norm on $V_{p}$. If $\psi_{\alpha, t} \circ u \in V_{p}$ for all $u$ in a dense subset of $V_{p}$, then $\psi_{\alpha, t} \circ u \in V_{p}$ for all $u \in V_{p}$; that is, Assumption 2.1 is satisfied.

Proof. From the definition of $\psi_{\alpha, t}$ we clearly have

$$
\left|\psi_{\alpha, t} \circ u\right| \leq t \alpha^{t-1}|u| \text {. }
$$

Also, by (3.4)

$$
\left|\nabla\left(\psi_{\alpha, t} \circ u\right)\right| \leq t \alpha^{t-1}|\nabla u| .
$$

Now assume that $W \subset V_{p}$ is a dense set such that $\psi_{\alpha, t} \circ u \in V_{p}$ for all $u \in W$. Hence by the definition of the norm (2.6) and the above,

$$
\left\|\psi_{\alpha, t} \circ u\right\|_{V_{p}} \leq t \alpha^{t-1}\|u\|_{V_{p}}
$$

for all $u \in W$. Given $u \in V_{p}$ there exist $u_{n} \in W$ such that $u_{n} \rightarrow u$ in $V_{p}$. Clearly $\psi_{\alpha, t}$ is Lipschitz with

$$
\left|\psi_{\alpha, t}(\xi)-\psi_{\alpha, t}(\eta)\right| \leq t \alpha^{t-1}|\xi-\eta|
$$

and thus $\left\|\psi_{\alpha, t} \circ u_{n}-\psi_{\alpha, t} \circ u\right\|_{p} \leq t \alpha^{t-1}\left\|u_{n}-u\right\|_{p}$ for all $n \in \mathbb{N}$. Hence $\psi_{\alpha, t} \circ u_{n} \rightarrow$ $\psi_{\alpha, t} \circ u$ in $L_{p}(\Omega)$. By (A.1) the sequence $\left(\psi_{\alpha, t} \circ u_{n}\right)$ is bounded in $V_{p}$, and therefore $\psi_{\alpha, t} \circ u_{n} \rightarrow \psi_{\alpha, t} \circ u$ weakly in $V_{p}$. In particular this implies that $\psi_{\alpha, t} \circ u \in V_{p}$.

We now apply the above to the standard examples. First, if $V_{p}=W_{p}^{1}(\Omega)$, then $\psi_{\alpha, t} \circ u \in W_{p}^{1}(\Omega)$ for all $u \in W_{p}^{1}(\Omega)$. This covers the examples of the Neumann problem and also the Robin problem on a Lipschitz domain.

Next we look at the Dirichlet problem. We know that $W:=C_{c}(\Omega) \cap W_{p}^{1}(\Omega)$ is dense in $\mathscr{\circ}_{p}^{1}(\Omega)$. From the above and the continuity of $\psi_{\alpha, t}$ we clearly have $\psi_{\alpha, t} \circ u \in \stackrel{\circ}{W}_{p}^{1}(\Omega)$ for all $u \in W$ and therefore for all $u \in \stackrel{\circ}{W}_{p}^{1}(\Omega)$ by the above proposition.

We finally consider the space $V_{p}:=W_{p, p}^{1}(\Omega, \partial \Omega)$ which, as defined in Section 4 , is the completion of the space

$$
W:=\left\{u \in W_{p}^{1}(\Omega) \cap C(\bar{\Omega}):\|u\|_{V_{p}}<\infty\right\}
$$

with respect to the norm

$$
\|u\|_{V_{p}}=\left(\|u\|_{W_{p}^{1}}^{p}+\left\|\left.u\right|_{\partial \Omega}\right\|_{L_{p}(\partial \Omega)}^{p}\right)^{1 / p} .
$$

Since $\left|\psi_{\alpha, t} \circ u\right| \leq t \alpha^{t-1}|u|$ on $\bar{\Omega}$, it follows that $\psi_{\alpha, t} \circ u \in W_{p, p}^{1}(\Omega, \partial \Omega)$ for all $u \in W$ and thus by definition of $W_{p, p}^{1}(\Omega, \partial \Omega)$ and by the above proposition for all $u \in W_{p, p}^{1}(\Omega, \partial \Omega)$.

\section{ACKNOWLEDGEMENT}

The first author is grateful for the kind hospitality of the Department of Mathematics, University of West Bohemia in Pilsen, and the second author for the kind hospitality of the School of Mathematics and Statistics, University of Sydney. 


\section{REFERENCES}

[1] Robert A. Adams, Sobolev spaces, Pure and Applied Mathematics, vol. 65, Academic Press, New York, 1975. MR 0450957 (56:9247)

[2] S. Agmon, A. Douglis, and L. Nirenberg, Estimates near the boundary for solutions of elliptic partial differential equations satisfying general boundary conditions. I, Comm. Pure Appl. Math. 12 (1959), 623-727. MR0125307 (23:A2610)

[3] Aomar Anane, Simplicité et isolation de la première valeur propre du p-laplacien avec poids, C. R. Acad. Sci. Paris Sér. I Math. 305 (1987), no. 16, 725-728. MR920052 (89e:35124)

[4] Jürgen Appell and Peter P. Zabrejko, Nonlinear superposition operators, Cambridge Tracts in Mathematics, vol. 95, Cambridge University Press, Cambridge, 1990. MR 1066204(91k:47168)

[5] Wolfgang Arendt and Mahamadi Warma, The Laplacian with Robin boundary conditions on arbitrary domains, Potential Anal. 19 (2003), no. 4, 341-363. MR1988110(2004d:31013)

[6] Daniel Daners, Robin boundary value problems on arbitrary domains, Trans. Amer. Math. Soc. 352 (2000), no. 9, 4207-4236. MR1650081 (2000m:35048)

[7] _ A priori estimates for solutions to elliptic equations on non-smooth domains, Proc. Roy. Soc. Edinburgh Sect. A 132 (2002), no. 4, 793-813. MR1926917 (2003g:35017)

[8] P. Drábek, Solvability and bifurcations of nonlinear equations, Pitman Research Notes in Mathematics Series, vol. 264, Longman Scientific \& Technical, Harlow, Essex, 1992. MR.1175397 (94e:47084)

[9] Pavel Drábek and Jaroslav Milota, Methods of nonlinear analysis, Birkhäuser Advanced Texts, Birkhäuser, Basel, 2007. MR2323436

[10] J. P. García Azorero and I. Peral Alonso, Existence and nonuniqueness for the p-Laplacian: nonlinear eigenvalues, Comm. Partial Differential Equations 12 (1987), no. 12, 1389-1430. MR912211 (89e:35058)

[11] David Gilbarg and Neil S. Trudinger, Elliptic partial differential equations of second order, Second, Grundlehren der Mathematischen Wissenschaften, vol. 224, Springer-Verlag, Berlin, 1983. MR0737190 (86c:35035)

[12] Gary M. Lieberman, Boundary regularity for solutions of degenerate elliptic equations, Nonlinear Anal. 12 (1988), no. 11, 1203-1219. MR969499 (90a:35098)

[13] Peter Lindqvist, On the equation $\operatorname{div}\left(|\nabla u|^{p-2} \nabla u\right)+\lambda|u|^{p-2} u=0$, Proc. Amer. Math. Soc. 109 (1990), no. 1, 157-164. MR.1007505 (90h:35088)

[14] V. G. Maz'ja, Classes of domains and imbedding theorems for function spaces, Soviet Math. Dokl. 1 (1960), 882-885. MR0126152 (23:A3448)

[15] Vladimir G. Maz'ja, Sobolev spaces, Springer Series in Soviet Mathematics, Springer-Verlag, Berlin, 1985. Translated from the Russian by T. O. Shaposhnikova. MR817985 (87g:46056)

[16] Jürgen Moser, A new proof of De Giorgi's theorem concerning the regularity problem for elliptic differential equations, Comm. Pure Appl. Math. 13 (1960), 457-468. MR0170091 $(30: 332)$

[17] Jindřich Nečas, Les méthodes directes en théorie des équations elliptiques, Academia, Prague, 1967. MR0227584(37:3168)

[18] Mike O'Leary, Integrability and boundedness of local solutions to doubly degenerate quasilinear parabolic equations, Adv. Differential Equations 5 (2000), no. 10-12, 1465-1492. MR:1785682 (2001h:35108)

[19] Mitsuharu Otani and Toshiaki Teshima, On the first eigenvalue of some quasilinear elliptic equations, Proc. Japan Acad. Ser. A Math. Sci. 64 (1988), no. 1, 8-10. MR953752 (89h:35257)

[20] Peter Tolksdorf, Regularity for a more general class of quasilinear elliptic equations, J. Differential Equations 51 (1984), no. 1, 126-150. MR727034 (85g:35047)

[21] Kôsaku Yosida, Functional analysis, 6th ed., Grundlehren der Mathematischen Wissenschaften, vol. 123, Springer-Verlag, Berlin, 1980. MR617913 (82i:46002)

School of Mathematics and Statistics, The University of Sydney, NSW 2006, AusTRALIA

E-mail address: D.Daners@maths.usyd.edu.au

Department of Mathematics, University of West Bohemia, P.O. Box 314, 30614 Pilsen, Czech Republic

E-mail address: pdrabek@kma.zcu.cz 